\title{
Zero-Free Intervals for Flow Polynomials of Near-Cubic Graphs
}

\author{
Bill Jackson \\ School of Mathematical Sciences, \\ Queen Mary, University of London, \\ Mile End Road, London E1 4NS, England
}

4 July 2005

\begin{abstract}
Let $P(G, t)$ and $F(G, t)$ denote the chromatic and flow polynomials of a graph $G$. G.D. Birkhoff and D.C. Lewis showed that, if $G$ is a plane near triangulation, then the only zeros of $P(G, t)$ in $(-\infty, 2]$ are 0,1 and 2 . We will extend their theorem by showing that a stonger result to the dual statement holds for both planar and non-planar graphs: if $G$ is a bridgeless graph with at most one vertex of degree other than three, then the only zeros of $F(G, t)$ in $(-\infty, \alpha]$ are 1 and 2 , where $\alpha \approx 2.225 \ldots$ is the real zero in $(2,3)$ of the polynomial $t^{4}-8 t^{3}+22 t^{2}-28 t+17$. In addition we construct a sequence of 'near-cubic' graphs whose flow polynomials have zeros converging to $\alpha$ from above.
\end{abstract}

\section{Introduction}

All graphs considered are finite and may contain loops and multiple edges. We shall refer to graphs without loops or multiple edges as simple graphs. We use $P(G, t)$ to denote the chromatic polynomial of a graph $G$, and $F(G, t)$ to denote its flow polynomial. We shall use the terms chromatic root and flow root of $G$ to refer to the zeros of $P(G, t)$ and $F(G, t)$, respectively. The study of the distribution of chromatic roots was initiated by G.D. Birkhoff and D.C. Lewis in [1]. Inspired by the 4-Colour Conjecture, they showed that for all plane near triangulations $G$, $P(G, t)$ has no zeros in the intervals $(-\infty, 0),(0,1),(1,2)$ and $[5, \infty)$. In addition, D.R. Woodall [15] has shown that 2 is a simple zero of $P(G, t)$ if $G$ is 3-connected.

For an arbitrary loopless graph $G$, it is known, see Tutte [12] or Woodall [15], that $P(G, t)$ has no zeros in the intervals $(-\infty, 0)$ and $(0,1)$, that 0 is a zero of 
$P(G, t)$ of multiplicity equal to the number of components of $G$, and that 1 is a zero of $P(G, t)$ of multiplicity equal to the number of non-trivial blocks of $G$, where a block is non-trivial if it has at least one edge. In addition, in [3], it is shown that $P(G, t)$ has no zeros in the interval $\left(1, \frac{32}{27}\right]$. Wakelin [13] showed that the dual statements also hold for an arbitrary bridgeless graph $G$ : $F(G, t)$ has no zeros in the intervals $(-\infty, 1)$ and $\left(1, \frac{32}{27}\right]$ and 1 is a zero of $F(G, t)$ of multiplicity equal to the number of non-trivial blocks of $G$. (A common extension of these results on chromatic and flow polynomials to matroids is obtained in [2].)

In this paper we show that the dual statements to the above mentioned results on plane near triangulations hold for both planar and non-planar graphs. We will consider near-cubic graphs: graphs with minimum degree at least two and at most one vertex of degree greater then three. We will show that if $G$ is bridgeless and near-cubic, then $F(G, t)$ has no zeros in $(1,2) ; 2$ is a simple zero of $F(G, t)$ when $G$ is 3-connected; and $F(G, t)$ has no zeros in $(2, \alpha]$, where $\alpha \approx 2.225 \ldots$ is the real zero in $(2,3)$ of the polynomial $t^{4}-8 t^{3}+22 t^{2}-28 t+17$. We give an example of a sequence of near-cubic graphs whose flow polynomials have zeros converging to $\alpha$ from above. These results extend the above mentioned results on chromatic roots of plane near triangulations in $(\infty, 2]$ by planar duality. We refer the reader to [4] for a more detailed survey of chromatic and flow roots of graphs.

\section{Definitions and Preliminary Results}

Let $G$ be a graph. An edge-cut of $G$ is the set of edges, $S$, from $U$ to $V(G)-U$ for some proper subset $U \subset V(G)$. We say that the subgraphs of $G$ induced by $U$ and $V(G)-U$ are the sides of $S$ and that $S$ is a $k$-edge-cut if $|S|=k$. A bridge of $G$ is a 1-edge-cut. The graph $G$ is $k$-edge-connected if $G$ has no $r$-edge-cuts for $r<k$. An edge-cut $S$ of $G$ is cyclic if both sides of $S$ contain circuits, and $G$ is cyclically $k$-edge-connected if each cyclic edge-cut of $G$ has at least $k$ edges.

We say that $G$ is $k$-connected for some integer $k \geq 2$ if $G$ is loopless, $|V(G)| \geq$ $k+1$, and $G-U$ is connected for all all $U \subset V(G)$ with $|U|<k$. A vertex $x$ is a cut-vertex of a graph $G$ if $G-x$ has more components than $G$. We say that $G$ is non-separable if either $|V(G)|=1$ and $|E(G)| \leq 1$, or $G$ is loopless, connected and has no cut-vertices. Thus a graph on at least three vertices is non-separable if and only if it is 2-connected. A block of $G$ is a maximal non-separable subgraph of $G$. (Thus every loop of $G$ is the edge set of a block of $G$.)

Given an edge $e$ of $G$, we shall use $G / e$ and $G-e$ to denote the graphs obtained from $G$ by contracting $e$ and deleting $e$, respectively. Note that, if $e$ is a loop, then $G / e=G-e$ and that, if $e$ belongs to a set of parallel edges, then every edge in the set other than $e$ becomes a loop in $G / e$. Throughout this paper we use $n$ and $m$ 
to denote the numbers of vertices and edges, respectively, in a graph $G$. We shall extend this notation by using subscripts and superscripts. Thus, for example, the number of edges in a graph $G_{1}^{+}$will be denoted by $m_{1}^{+}$.

\section{Flow Polynomials}

Let $\Gamma$ be an additive abelian group and $G$ be a graph. Suppose we construct a digraph $\vec{G}$ by giving the edges of $G$ an arbitrary orientation. For $U \subseteq V(G)$ and $\bar{U}=V(G)-U$, let $E^{+}(U)$ be the set of arcs from $U$ to $\bar{U}$ in $\vec{G}$ and $E^{-}(U)=E^{+}(\bar{U})$. Let $f: E(\vec{G}) \rightarrow \Gamma$ and put $f^{+}(U)=\sum_{e \in E^{+}(U)} f(e)$ and $f^{-}(U)=\sum_{e \in E^{-}(U)} f(e)$. For $v \in V(G)$ let $f^{+}(v)=f^{+}(\{v\})$ and $f^{-}(v)=f^{-}(\{v\})$. Then $f$ is a $\Gamma$-flow for $G$, with respect to $\vec{G}$, if $f^{+}(v)=f^{-}(v)$ for all $v \in V(G)$. If, in addition, $f(e) \neq 0$ for all $e \in E(G)$, then we say that $f$ is a nowhere-zero $\Gamma$-flow for $G$. It can be seen that the condition $f^{+}(v)=f^{-}(v)$ for all $v \in V(G)$ is equivalent to the apparently stronger condition that $f^{+}(U)=f^{-}(U)$ for all $U \subseteq V(G)$. Thus, if $G$ has a nowhere-zero $\Gamma$-flow, then $G$ is bridgeless. Since reversing the orientation on an edge $e$ of $\vec{G}$ is equivalent to replacing $f(e)$ by $-f(e)$, the number of distinct nowhere-zero $\Gamma$ flows for $G$ is independent of the chosen orientation $\vec{G}$ of $G$.

A nowhere-zero $t$-flow for $G$ is a nowhere-zero $\mathbb{Z}$-flow, $f$, such that $|f(e)| \leq t-$ 1 for all $e \in E(G)$. Tutte [9] has shown that $G$ has a nowhere-zero $t$-flow if and only if $G$ has a nowhere-zero $\mathbb{Z}_{t}$-flow. Furthermore, the number of distinct nowhere-zero $\Gamma$-flows for $G$ is the same for all abelian groups $\Gamma$ of the same order. Note, however, that the number of nowhere-zero $\mathbb{Z}_{t}$-flows for $G$ may differ from the number of nowhere-zero $t$-flows for $G$. Nowhere-zero flows were introduced by Tutte [9] as a dual concept to proper colourings. He showed that a connected plane graph $G$ has a proper $t$-colouring if and only if its planar dual $G^{*}$ has a nowhere-zero $t$ flow. The two concepts differ for non-planar graphs, however. Indeed, whereas there exist loopless graphs which are not $t$-colourable for arbitrarily large integers $t$, Tutte [10] conjectured that every bridgeless graph has a nowhere-zero 5-flow. Jaeger [6] obtained a partial solution by showing that every bridgeless graph has a nowhere-zero 8-flow. His result was subsequently improved by Seymour:

Theorem 1 [8] Every bridgeless graph has a nowhere-zero 6-flow.

Following Tutte [10] we define the flow polynomial $F(G, t)$ of $G$ as the number of distinct nowhere-zero $\mathbb{Z}_{t}$-flows for $G$ for any positive integer $t$. Thus $F(G, t) \equiv 1$ if $E(G)=\emptyset$ and $F(G, t) \equiv 0$ if $G$ has a bridge.

By the above remarks, $F(G, t)$ is independent of the chosen orientation of $G$. Note also that since the existence of a nowhere-zero $t$-flow for $G$ implies the existence of a nowhere-zero $(t+1)$-flow by definition, and is equivalent to the existence 
of a nowhere-zero $\mathbb{Z}_{t}$-flow as noted above, we may deduce that if $F\left(G, t_{0}\right) \neq 0$ for some positive integer $t_{0}$, then $F(G, t) \neq 0$ for all integers $t \geq t_{0}$.

We could also consider the polynomial $I(G, t)$ defined to be the number of distinct nowhere-zero $t$-flows for $G$. Kochal [7] gives relationships between $I(G, t)$ and $F(G, t)$, but these seem to be the only results on $I(G, t)$ in the literature. Attention has concentrated on $F(G, t)$ because it is dual to $P(G, t)$ for plane graphs: if $G$ is a connected plane graph and $G^{*}$ its planar dual, then there is a surjection from the $t$-vertex-colourings of $G^{*}$ to the nowhere-zero $\mathbb{Z}_{t}$-flows for $G$ such that each nowhere-zero $\mathbb{Z}_{t}$-flow for $G$ has exactly $t$ pre-images, see [10]. Thus

$$
F(G, t)=t^{-1} P\left(G^{*}, t\right) .
$$

We may use this identity to restate results and conjectures on chromatic roots of families of plane graphs in terms of flow roots of the dual families. For non-planar graphs, however, the zero distributions of chromatic and flow polynomials are very different. Indeed there is a tendency for the zero distribution of flow polynomials to be similar in both the planar and non-planar case. The results of this paper are an example of this. The following conjectured strengthening of Tutte's 5-flow conjecture due to Welsh [14] would be another example.

Conjecture 1 Let $G$ be a bridgeless graph. Then $F(G, t)>0$ for all $t \in(4, \infty)$.

Although Theorem 1 implies that $F(G, t)>0$ for all bridgeless graphs $G$ and all integers $t \geq 6$, it is not even known whether there exists a finite $t_{0}$ such that $F(G, t)>0$ for all $t \in\left(t_{0}, \infty\right)$. This contrasts with the result of Birkoff and Lewis [1] that $P(G, t)>0$ for all loopless planar graphs $G$ and all $t \in[5, \infty)$, and their conjecture that $P(G, t)>0$ for all $t \in[4, \infty)$.

\section{Some recurrence relations for flow polynomials}

Our inductive proof technique for working with flow polynomials is based on the following elementary recurrence relations.

Lemma 2 Let $G$ be a graph and $e$ be an edge of $G$.

(a) If $e$ is a loop then $F(G, t)=(t-1) F(G / e, t)$.

(b) If e is not a loop then $F(G, t)=F(G / e, t)-F(G-e, t)$.

Proof. We may deduce that the lemma holds for each integer $t \geq 2$, and hence for all $t$, as follows. If $e$ is a loop then each nowhere-zero $\mathbb{Z}_{t}$-flow for $G-e$ corresponds to $t-1$ nowhere-zero $\mathbb{Z}_{t}$-flows for $G$. If $e$ is not a loop then each nowhere-zero $\mathbb{Z}_{t^{-}}$ flow for $G / e$ coresponds to either a nowhere-zero $\mathbb{Z}_{t}$-flow for $G$ or a nowhere-zero $\mathbb{Z}_{t}$-flow for $G-e$. 
We may use Lemma 2 inductively to obtain the following reduction lemmas for flow polynomials of graphs of low connectivity.

Lemma 3 The flow polynomials of two homeomorphic graphs are identical.

Lemma 4 Let $G$ be a graph and $G_{1}$ and $G_{2}$ be edge-disjoint subgraphs of $G$ such that $G_{1} \cup G_{2}=G$ and $\left|V\left(G_{1}\right) \cap V\left(G_{2}\right)\right| \leq 1$. Then

$$
F(G, t)=F\left(G_{1}, t\right) F\left(G_{2}, t\right) .
$$

Lemma 5 Let $G$ be a graph, $v$ be a vertex of $G, e=u_{1} u_{2}$ be an edge of $G$, and $H_{1}$ and $H_{2}$ be edge-disjoint subgraphs of $G$ such that $H_{1} \cup H_{2}=G-e, H_{1} \cap H_{2}=\{v\}$, $u_{1} \in V\left(H_{1}\right)$ and $u_{2} \in V\left(H_{2}\right)$. Let $G_{i}$ be obtained from $G$ by contracting $E\left(H_{3-i}\right)$, for $i \in\{1,2\}$. Then

$$
F(G, t)=\frac{F\left(G_{1}, t\right) F\left(G_{2}, t\right)}{(t-1)} .
$$

As a special case of Lemma 5 we have

Lemma 6 Let $G$ be a graph, $S$ be a 2-edge-cut of $G$, and $H_{1}$ and $H_{2}$ be the sides of $S$. Let $G_{i}$ be obtained from $G$ by contracting $E\left(H_{3-i}\right)$, for $i \in\{1,2\}$. Then

$$
F(G, t)=\frac{F\left(G_{1}, t\right) F\left(G_{2}, t\right)}{(t-1)} .
$$

Lemma 7 Let $G$ be a graph, $S$ be a 3-edge-cut of $G$, and $H_{1}$ and $H_{2}$ be the sides of $S$. Let $G_{i}$ be obtained from $G$ by contracting $E\left(H_{3-i}\right)$, for $i \in\{1,2\}$. Then

$$
F(G, t)=\frac{F\left(G_{1}, t\right) F\left(G_{2}, t\right)}{(t-1)(t-2)} .
$$

Lemma 8 Let $G$ be a graph, $x$ be a vertex of $G, e=u_{1} u_{2}, f=v_{1} v_{2}$ be edges of $G$, and $H_{1}$ and $H_{2}$ be edge-disjoint subgraphs of $G$ such that $H_{1} \cup H_{2}=G-\{e, f\}$, $H_{1} \cap H_{2}=\{x\}, u_{1}, v_{1} \in V\left(H_{1}\right)$ and $u_{2}, v_{2} \in V\left(H_{2}\right)$. Let $G^{+}$be the graph obtained from $G-x$ by adding two new vertices, $x_{1}, x_{2}$, an edge $x_{1} x_{2}$, and an edge from $x_{i}$ to each of the neighbours of $x$ in $H_{i}$, for $1 \leq i \leq 2$. For $i=1,2$, let $G_{i}^{+}$be the graph obtained from $G^{+}\left[V\left(H_{i}\right)-x+x_{i}\right]$ by adding a new vertex $y_{i}$ and edges $y_{i} x_{i}, y_{i} u_{i}, y_{i} v_{i}$. Let $G_{i}^{-}=G_{i}^{+}-x_{i} y_{i}$. Then

$$
(t-1)(t-2) F(G, t)=F\left(G_{1}^{+}, t\right) F\left(G_{2}^{+}, t\right)+(t-2) F\left(G_{1}^{-}, t\right) F\left(G_{2}^{-}, t\right) .
$$

Proof. By Lemma 2, $F(G, t)=F\left(G^{+}, t\right)+F\left(G^{-}, t\right)$. The lemma now follows by applying Lemmas 6 and 7 to $G^{-}$and $G^{+}$, respectively. 


\section{Near-cubic graphs}

The family of cubic graphs has special significance for nowhere-zero flows since many problems on flows in general graphs can be reduced to the special case of cubic graphs. In particular, the truth of Tutte's 5-flow conjecture and Conjecture 1 would follow from their special cases for cubic graphs. Since the family of cubic graphs is not closed under the operations of edge contraction or deletion, it is often helpful to consider the larger family of near-cubic graphs. We shall use the ordered pair $(G, x)$ to represent a near-cubic graph $G$ together with a distinguished vertex $x$ such that each vertex of $G$ other than $x$ has degree two or three. A $k$-bond is a loopless graph with two vertices and $k$ edges. We denote the isomorphism class of all $k$-bonds by $K_{2}^{k}$.

\subsection{The interval $(-\infty, 2)$}

We show in this subsection that a bridgeless near-cubic graph $(G, x)$ has no flow roots in $(-\infty, 2)$ apart from the integer flow root at 1 . We shall in fact prove a stronger result concerning the derivatives of the quotient polynomial $q_{1}(G, t)=$ $F(G, t) /(t-1)$. Since $G$ is near-cubic, it has at least one edge and hence 1 is a zero of $F(G, t)$. Since $F(G, t)$ is a polynomial in $t$ of degree $m-n+1$, it follows that $q_{1}(G, t)$ is a polynomial of degree $m-n$. Let $q_{1}^{(i)}(G, t)$ be the $i^{\prime}$ th derivative of $q_{1}(G, t)$.

Theorem 9 Let $(G, x)$ be a non-separable near-cubic graph with $n$ vertices and $m$ edges. Then $q_{1}^{(i)}(G, t)$ is non-zero with sign $(-1)^{m-n+i}$ for all $0 \leq i \leq m-n$ and all $t \in(-\infty, 2)$.

Proof. We proceed by contradiction. Suppose the theorem is false and let $(G, x)$ be a counterexample chosen such that $m$ is as small as possible. If $n=1$ then $m=1$, $F(G, t)=t-1$ and $q_{1}(G, t)=1$. If $n=2$ then either $G=K_{2}^{2}, F(G, t)=t-1$ and $q_{1}(G, t)=1$, or $G=K_{2}^{3}, F(G, t)=(t-1)(t-2)$ and $q_{1}(G, t)=t-2$. Since the theorem holds in all three cases we have $n \geq 3$. Thus $G$ is 2 -connected. Using Lemma 3 we may also deduce that $G$ has minimum degree three.

Claim $1 G-x$ is 2-edge-connected.

Proof. Since $G$ is non-separable, $G-x$ is connected. Suppose $G-x$ has a bridge $e$. Adopting the notation of Lemma 5, we have $F(G, t)=F\left(G_{1}, t\right) F\left(G_{2}, t\right) /(t-1)$, and hence $q_{1}(G, t)=q_{1}\left(G_{1}, t\right) q_{1}\left(G_{2}, t\right)$. Thus

$$
q_{1}^{(i)}(G, t)=\sum_{j=0}^{i}\left(\begin{array}{l}
i \\
j
\end{array}\right) q_{1}^{(j)}\left(G_{1}, t\right) q_{1}^{(i-j)}\left(G_{2}, t\right) .
$$


The graphs $\left(G_{1}, x\right)$ and $\left(G_{2}, x\right)$ are both non-separable near-cubic graphs with fewer edges than $G$. Since $m=m_{1}+m_{2}-1$ and $n=n_{1}+n_{2}-1$, we may deduce that the theorem holds for $G$ by applying induction to $G_{1}$ and $G_{2}$.

Let $e$ be an edge of $G$ incident to $x$. The facts that $(G, x)$ is near-cubic, $G-x$ is 2-edge-connected and $n \geq 3$ imply that $(G / e, x)$ and $(G-e, x)$ are non-separable near-cubic graphs. Applying Lemma 2 to $G-e$ and $G / e$, and differentiating $i$ times, we may deduce inductively that the theorem holds for $G$.

Note that if $(G, x)$ is a separable bridgeless near-cubic graph, then $x$ is the unique cut-vertex of $G$ and we may apply Theorem 9 to each block of $G$ using Lemma 4 to deduce that $q_{1}^{i}(G, t) \neq 0$ for all $0 \leq i \leq m-n$ and all $t \in(-\infty, 2)$.

\subsection{The mutiplicity of the zero at 2}

It is easy to see that a graph $G$ has a nowhere-zero $\mathbb{Z}_{2}$-flow if and only if every vertex of $G$ has even degree. Thus 2 is a zero of $F(G, t)$ if and only if $G$ has at least one vertex of odd degree. We shall show in this section that 2 is a simple zero of a cubic graph $G$ if and only if $G$ is 3-connected. As in the previous section, our inductive proof requires us to work with near-cubic graphs.

We say that a graph is essentially 3-connected if it is a subdivision of a 3connected graph or a $k$-bond for some $k \geq 3$. We shall use the following 'reduction' lemma due to Tutte [11, Theorem 12.65].

Lemma 10 Let $G$ be a 3-connected graph and $e \in E(G)$. Then either $G-e$ is essentially 3-connected or G/e is 3-connected.

Theorem 11 Let $(G, x)$ be a non-separable near-cubic graph with $m$ edges, $n$ vertices, and at least one vertex of degree three. Let

$$
q_{2}(G, t)=\frac{F(G, t)}{(t-1)(t-2)} .
$$

Then $q_{2}(G, t)$ is a polynomial in $t$. Furthermore:

(a) if $G$ is not essentially 3-connected then $q_{2}(G, 2)=0$;

(b) if $G$ is essentially 3-connected then $q_{2}(G, 2)$ is non-zero with sign $(-1)^{m-n+1}$.

Proof. The fact that $q_{2}(G, t)$ is a polynomial in $t$ follows from the fact that $F(G, t)$ is a polynomial in $t$ with a zero at 1 and a zero at 2 (since $G$ has at least one vertex of degree three).

(a) Suppose that $G$ is not essentially 3 -connected. Since $(G, x)$ is near cubic it follows that we can find a vertex $v$ of $G$, an edge $e$ of $G$, and edge-disjoint subgraphs 
$H_{1}$ and $H_{2}$ of $G$ such that $H_{1} \cup H_{2}=G-e, H_{1} \cap H_{2}=\{v\}$, and both $H_{1}$ and $H_{2}$ contain a vertex of degree three in $G$. Without loss of generality $x \in V\left(H_{1}\right)$. Let $G_{1}$ be obtained from $G$ by contracting $E\left(H_{2}\right)$ to $v$, and define $G_{2}$ analogously. By Lemma $5, F(G, t)=F\left(G_{1}, t\right) F\left(G_{2}, t\right) /(t-1)$, and hence

$$
q_{2}(G, t)=q_{2}\left(G_{1}, t\right) q_{2}\left(G_{2}, t\right)(t-2) .
$$

It can be seen that $\left(G_{1}, x\right)$ and $\left(G_{2}, v\right)$ are both non-separable near-cubic graphs with fewer edges than $G$ and both contain a vertex of degree three. Thus $q_{2}\left(G_{1}, t\right)$ and $q_{2}\left(G_{2}, t\right)$ are both polynomials in $t$ and $q_{2}(G, 2)=0$ by (1). This completes the proof of (a).

(b) Suppose that $G$ is essentially 3-connected. We prove (b) by induction on $m$. Let $G^{\prime}$ be the graph of minimum degree three which is homeomorphic to $G$. If $G^{\prime} \neq G$ then since $q_{2}\left(G^{\prime}, t\right)=q_{2}(G, t)$ by Lemma 3 , and $m^{\prime}-n^{\prime}=m-n$, we may deduce inductively that the theorem holds for $G$. Thus we may suppose that $G^{\prime}=G$. If $G=K_{2}^{3}$, then $F(G, t)=(t-1)(t-2), q_{2}(G, t) \equiv 1$ and the theorem holds for $G$. Hence we may assume that $G$ is 3 -connected.

Let $e$ be an edge of $G$ incident to $x$. By Lemma 10, either $G-e$ or $G / e$ is essentially 3 -connected. If $G-e$ is essentially 3 -connected, then we may use Lemma 2 to deduce that (b) holds for $G$ by applying (b) inductively to $G-e$, applying (a) to $G / e$ if $G / e$ is not essentially 3-connected, and applying (b) inductively to $G / e$ if $G / e$ is essentially 3 -connected. A similar proof holds if $G / e$ is essentially 3 -connected

\subsection{Cleavage units}

Tutte [11] developed a theory by which a 2-connected graph $G$ can be uniquely decomposed into pieces called cleavage units which are either 3-connected simple graphs or bonds or circuits. We will use this decomposition extensively in the next subsection to show that the interval $(2, \alpha]$ contains no flow roots of nearcubic graphs. We give a brief description of Tutte's theory below for the sake of completeness.

We shall adopt the notation and terminology of Tutte [11] with the exception that we will refer to 'polygons', 'simple paths', and 'valency' as circuits, paths and degree respectively. Let $x$ and $y$ be vertices of a 2-connected graph $G$ such that $G-\{x, y\}$ has components $G_{1}, G_{2}, \ldots, G_{r}$, with $r \geq 2$ if $x y$ is not a multiple edge of $G$, and $r \geq 1$ otherwise. Each of the subgraphs of $G$ induced by $V\left(G_{i}\right) \cup\{x, y\}$, but with edges joining $x$ and $y$ deleted, is called an $\{x, y\}$-component of $G$. In addition, if $x y \in E(G)$, the subgraph induced by $\{x, y\}$ is a trivial $\{x, y\}$-component of $G$. Let $H$ be an $\{x, y\}$-component of $G$ and put $H^{\prime}=G-(H-\{x, y\})$. We say that $H$ 
is excisable if $H$ is nontrivial and either $H$ or $H^{\prime}$ is a 2-connected graph or a $k$-bond for some $k \geq 2$. If an excisable $\{x, y\}$-component $H$ of $G$ exists, we say that $\{x, y\}$ is a hinge of $G$ and $H$ is called a hinge component of $G$. A hinge $\{x, y\}$ of $G$ is of Type $I$ if $G$ has exactly two $\{x, y\}$-components and of Type II otherwise.

For each hinge $\{x, y\}$ of $G$ we adjoin to $G$ a virtual edge with ends $x$ and $y$ for each excisable $\{x, y\}$-component of $G$ to form the augmented graph $G^{a}$. Two distinct hinge components of $G$ give rise to the same virtual edge if and only if they are the two $\{x, y\}$-components of some hinge $\{x, y\}$ of Type I. The virtual edges are required to be distinct from the true edges of $G$ (but may be parallel to them). If $H$ is an excisable $\{x, y\}$-component of $\mathrm{G}$, the two graphs $D_{1}$ and $D_{2}$ derived from $H$ and $H^{\prime}$ by adjoining to each of $H$ and $H^{\prime}$ the virtual edge $e$ associated with $H$ are called the cleavage graphs of $G$ at $e$.

The cleavage units of $G$ are the minimal cleavage graphs obtained by recursively constructing cleavage graphs from cleavage graphs. Thus no cleavage unit of $G$ can have a hinge, and each virtual edge of $G$ belongs to exactly two cleavage units. The cleavage unit tree $T$ of $G$ is the graph whose vertices and edges are the cleavage units and virtual edges, respectively, of $G$, in which a cleavage unit $D$ and an edge $e$ are incident in $T$ if and only if $e$ is an edge of $D$. We say that $G$ is a cleavage unit path if its cleavage unit tree is a path on at least two vertices.

These definitions are illustrated for the graph $G$ in Figure 1 . We form $G^{a}$ by adding the five virtual edges $e_{1}, e_{2}, e_{3}, e_{4}$ and $e_{5}$ to $G$. The hinges of $G$ are the pairs of end vertices of the virtual edges. The cleavage units of $G, X_{1}, X_{2}, X_{3}, X_{4}, X_{5}, X_{6}$, and its cleavage unit tree, $T$, are shown in Figure 2.

Tutte shows in [11, Chapter 11] that the cleavage unit tree of a 2-connected graph $G$ is indeed a tree and that each cleavage unit of $G$ is either a 3-connected simple graph or a circuit of length at least three, or a bond with at least three edges. By an end-cleavage unit of $G$ we shall mean a cleavage unit which has degree one in the cleavage unit tree. By an internal vertex of $G$ we shall mean a vertex which does not belong to any hinge of $G$. Thus $v$ is an internal vertex of $G$ if either $d(v)=2$, or $G-v$ is non-separable and $v$ is not incident with a parallel edge of $G$.

We shall need the following observations on the cleavage unit decomposition of a graph.

Lemma 12 Let $G$ be a 2-connected graph and $T$ be the cleavage unit tree of $G$.

(a) The set of circuit cleavage units and the set of bond cleavage units of $G$ are independent sets of vertices in $T$;

(b) Suppose $G$ has at least two end cleavage units. Let $G^{\prime}$ be obtained from $G$ by adding a new vertex $x$ and an edge from $x$ to an internal vertex in each end cleavage unit of $G$. Then $G^{\prime}$ is essentially 3-connected. 

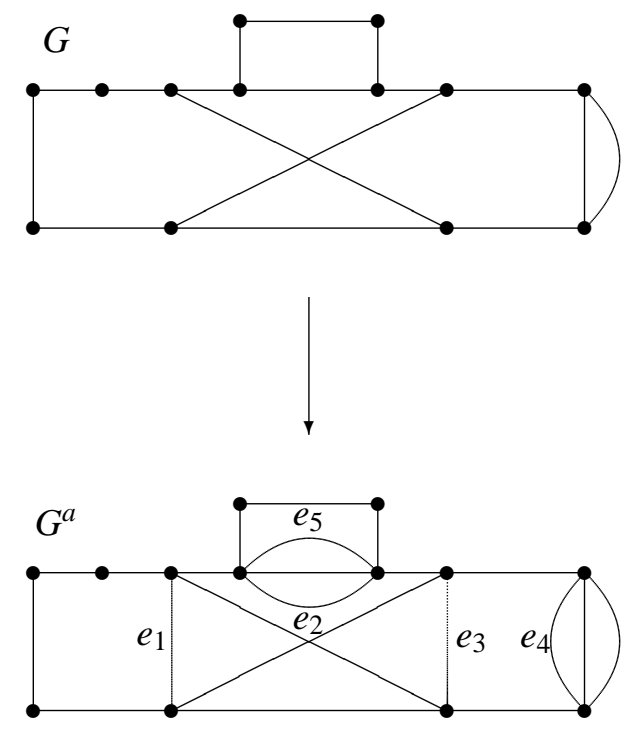

Figure 1: A graph $G$ and its augmented graph $G^{a}$. 


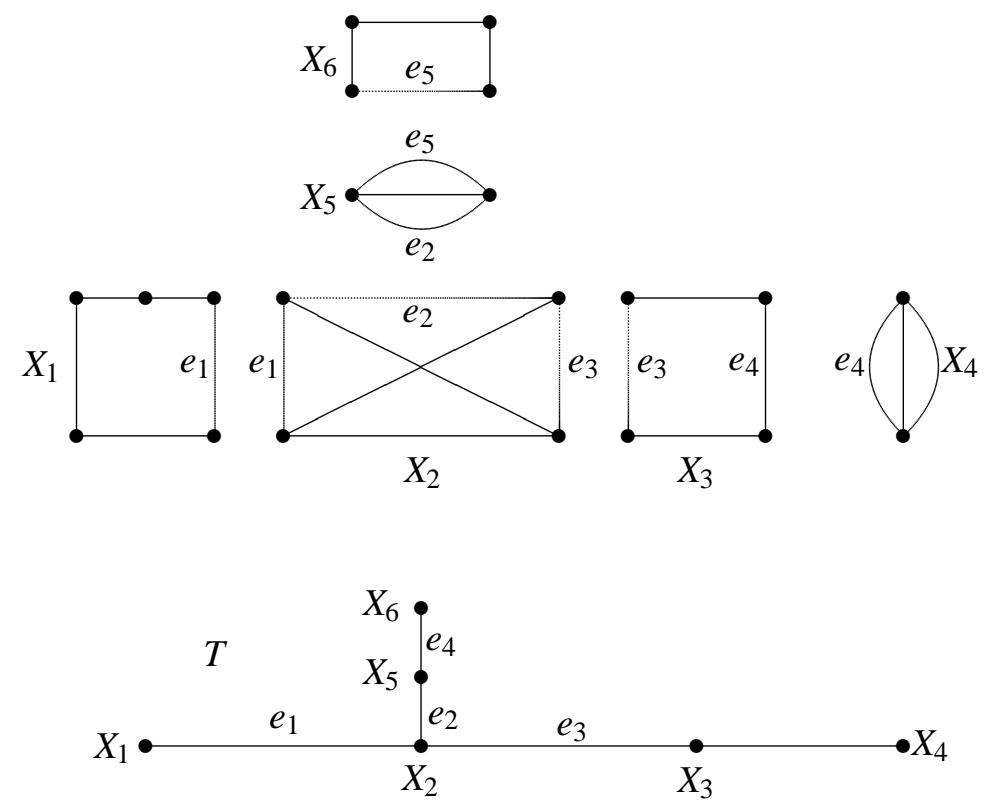

Figure 2: The cleavage units of $G$ and its cleavage unit tree $T$. 
We shall also need the following elementary result on 3-connectivity.

Lemma 13 Let $G$ be a 3-connected graph and $e=u v$ be an edge of $G$ of multiplicity one. Suppose $G-\{u, v\}$ is 2-connected. Then G/e is 3-connected.

\section{Cleavage units of near-cubic graphs}

It can be seen that the degree of a vertex in a cleavage unit of a 2-connected graph $G$ is bounded above by its degree in $G$. Thus the cleavage units of a near-cubic graph $(G, x)$ are themselves near-cubic. Lemma 5 can be used to express $F(G, t)$ in terms of the flow polynomials of its non-circuit cleavage units.

Lemma 14 Let $(G, x)$ be a 2-connected near-cubic graph and $G_{1}, G_{2}, \ldots, G_{c}$ be the cleavage units of $G$ which are not circuits. Then

$$
F(G, t)=(t-1)^{-c+1} \prod_{i=1}^{c} F\left(G_{i}, t\right) .
$$

Proof. We use induction on the total number of cleavage units of $G$. The lemma holds trivially if $G$ is a 3-connected simple graph, or a 3-bond, or a circuit (in the case of a circuit we have $c=0$, the empty product is equal to 1 , and $F(G, t)=$ $t-1)$. Thus we may assume that $G$ has at least two cleavage units and hence $G$ has a hinge $\{u, v\}$. Let $H$ be an excisable $\{u, v\}$-component, $e$ be the virtual edge incident with $u, v$ corresponding to $H$, and $G_{1}, G_{2}$ the cleavage graphs of $G$ at $e$. Suppose $u \neq x$. Then $d_{G}(u)=3$ and $u$ is incident to a bridge $u u^{\prime}$ of $G-v$. Relabelling if necessary we have $u^{\prime} \in V\left(G_{2}\right)$ and $d_{G_{2}}(u)=2$. By Lemmas 3 and $5, F(G, t)=(t-1)^{-1} F\left(G_{1}, t\right) F\left(G_{2}, t\right)$. Furthermore, each cleavage unit of $G$ is a cleavage unit of $G_{1}$ or $G_{2}$ (but not both). The lemma now follows by applying induction to $G_{1}$ and $G_{2}$.

As an example, we may apply Lemma 14 to the graph $G$ in Figure 1 to deduce that

$$
F(G, t)=(t-1)^{-2} F\left(K_{4}, t\right) F\left(K_{2}^{3}, t\right)^{2}=(t-1)(t-2)^{3}(t-3) .
$$

Lemma 14 and Theorem 11 imply:

Corollary 15 Let $(G, x)$ be a 2-connected near-cubic graph with at least one vertex of degree three. Then 2 is a zero of $F(G, t)$ of multiplicity $c$, where $c$ is the number of cleavage units of $G$ which are not circuits.

We shall use the following property of circuit cleavage units in graphs of maximum degree three. 
Lemma 16 Let $G$ be a 2-connected graph of maximum degree three. If $X$ is a circuit cleavage unit of $G$ then the virtual edges of $G$ contained in $X$ are independent.

Proof. Let $e=u v$ be a virtual edge of $G$ contained in $X$ and let $Y$ be the other cleavage unit of $G$ which contains $e$. Let $H_{1}, H_{2}$ be the cleavage graphs of $G$ at $e$. Realbelling if necessary we have $V(X) \subset H_{1}$ and $V(Y) \subseteq H_{2}$. Lemma 12(a) and the fact that $X$ is a circuit cleavage unit imply that $Y$ is not a circuit cleavage unit. Thus $d_{H_{2}}(u) \geq 3$ and $d_{H_{2}}(v) \geq 3$. Since $G$ has maximum degree three, we must have $d_{H_{1}}(u)=2=d_{H_{1}}(v)$. Thus $u$ and $v$ are both internal vertices of $H_{1}$. Hence neither $u$ nor $v$ can be incident to a virtual edge of $H_{1}$. Thus $e$ is the only virtual edge of $G$ incident to $u$ and $v$ in $X$.

\subsection{The interval $(2, \alpha]$}

We shall first construct a sequence of near-cubic graphs with flow roots converging to $\alpha$ from above. We then show that bridgeless near-cubic graphs have no flow roots in $(2, \alpha]$.

\subsubsection{A special family of near-cubic graphs}

Let $(H, x)$ and $(G, x)$ be near-cubic graphs, and $x u, u v, u w \in E(H)$. We say that $G$ is a single edge extension of $H$ if $G$ can be obtained from $H-u$ by adding four new vertices $u_{1}, u_{2}, u_{3}, u_{4}$ and eight new edges $x u_{1}, x u_{2}, u_{1} u_{2}, u_{2} u_{3}, u_{3} u_{4}, u_{4} u_{1}$, $u_{3} v, u_{4} w$. We say that $(G, x)$ is an extension of $(H, x)$ if $(G, x)$ can be obtained from $(H, x)$ by a sequence of single edge extensions. We shall show that near-cubic graphs which are extensions of $\left(K_{4}, x\right)$ have no flow roots in $(2, \alpha]$, but can have flow roots arbitrarily close to $\alpha$. An example of an extension of $\left(K_{4}, x\right)$ is given in Figure 3. This can be seen by recursively performing the inverse operation to a single edge extension: choosing a pair of edges $e_{1}, e_{2}$ incident to $x$ which belong to both a 3 -cycle $e_{1}, e_{2}, f_{0}$ and a 5 -cycle $e_{1}, e_{2}, f_{1}, f_{2}, f_{3}$; contracting $f_{0}, f_{1}, f_{2}, f_{3}$ and deleting either $e_{1}$ or $e_{2}$.

We need to consider one other family of near-cubic graphs. Let $\left(L_{0}, x\right)$ be the loopless near-cubic graph with $V\left(L_{0}\right)=\{x, y, z\}$ in which $x$ has degree two and all other vertices have degree three. Let $\mathcal{L}(x, y)$ be the isomorphism class of all near-cubic graphs which are isomorphic to either $\left(L_{0}, x\right)$ or a graph which can be obtained recursively from $\left(L_{0}, x\right)$ by applying the single edge extension operation to edges which are incident to $x$ but not to $y$. Let $\left(L_{1}, x\right)$ be the near-cubic graph obtained from $\left(L_{0}, x\right)$ by applying the single edge extension operation to the edge $x z$. Thus $V\left(L_{1}\right)=\left\{x, z_{1}, z_{2}, z_{3}, z_{4}, y\right\}$ and $E\left(L_{1}\right)=\left\{x z_{1}, x z_{2}, z_{1} z_{2}, z_{2} z_{3}, z_{3} z_{4}, z_{4} z_{1}, z_{3} y, z_{4} y\right\}$. 


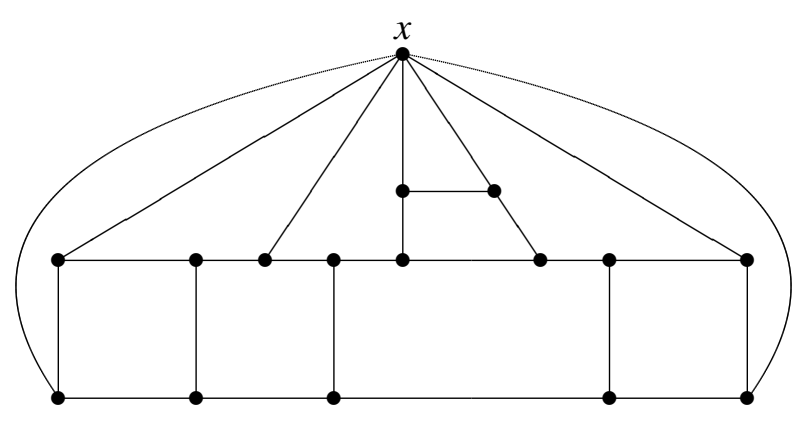

Figure 3: An extension of $\left(K_{4}, x\right)$

Lemma 17 Let $(G, x) \in \mathcal{L}(x, y)$. Let $t \in(2,3)$ and $g \geq 0$ be real numbers satisfying $f(t, g):=(3-t) g-2(t-2) \geq 0$ and

$h(t, g):=(3-t)^{2}\left(g^{2}+t-2\right)-(3-t)(t-2)(3-t+2 g)-g(t-2)\left(g^{2}+t-2\right) \geq 0$.

Then:

(a) $F(G-x y, t) \geq 0$ with strict inequality when $G \neq L_{0}$;

(b) $F(G, t) \geq g F(G-x y, t)$;

(c) $F(G, t)>0$.

Proof. We prove (a), (b) and (c) simultaneously by induction on $m$. If $m=4$ then $G=L_{0}, F(G, t)=(t-1)(t-2), F(G-x y, t) \equiv 0$ and the lemma holds. Hence we may suppose that $G$ is either equal to $\left(L_{1}, x\right)$ or can be obtained from $\left(L_{1}, x\right)$ by recursively applying the single edge extension operation to edges incident to $x$ but not $y$. Thus $d_{G}(y)=3, y x, y z_{3}, y z_{4}, z_{3} z_{4} \in E(G)$, and $G-\left\{x, y, z_{3}, z_{4}\right\}$ has a bridge $e=u_{1} u_{2}$ corresponding to the bridge $z_{1} z_{2}$ of $L_{1}-\left\{x, y, z_{3}, z_{4}\right\}$. Hence there exist $G_{1}, G_{2} \in \mathcal{L}(x, y)$, such that $G-\left\{y, z_{3}, z_{4}, e\right\}=\left(G_{1}-y\right) \cup\left(G_{2}-y\right),\left(G_{1}-y\right) \cap\left(G_{2}-\right.$ $y)=\{x\}, u_{1} \in V\left(G_{1}-y\right)$ and $u_{2} \in V\left(G_{2}-y\right)$. The near-cubic graphs $\left(G_{i}, x\right)$ are obtained by applying the same sequence of single edge extensions to the edge $x z$ of $\left(L_{0}, x\right)$ as were applied to the edge $x z_{i}$ in the construction of $(G, x)$ from $\left(K_{4}, x\right)$.

We shall express $F(G-x y, t)$ and $F(G, t)$ in terms of $F\left(G_{1}, t\right), F\left(G_{1}-x y, t\right)$, $F\left(G_{2}, t\right)$ and $F\left(G_{2}-x y, t\right)$. Let $G^{+}$be the graph obtained by contracting $\left\{z_{3} z_{4}, y z_{3}, y z_{4}\right\}$ 
onto $y$. Using Lemmas 6 and 8, we have

$$
\begin{aligned}
& (t-1) F(G-x y, t)=(t-1)(t-2) F\left(G^{+}-x y, t\right)= \\
& \quad=F\left(G_{1}, t\right) F\left(G_{2}, t\right)+(t-2) F\left(G_{1}-x y, t\right) F\left(G_{2}-x y, t\right) .
\end{aligned}
$$

Applying (a), (c) inductively to $G_{1}, G_{2}, G_{1}-x y, G_{2}-x y$ we deduce that $F(G-$ $x y, t)>0$. Hence (a) holds for $G$.

By Lemma 7, $(t-1) F(G, t)=(t-1)(t-3) F\left(G^{+}, t\right)$. Applying Lemma 2 to the edge $x y$ of $G^{+}$and then using Lemmas 5 and 8 , and the fact that $F\left(G_{i} / x y, t\right)=$ $F\left(G_{i}, t\right)+F\left(G_{i}-x y, t\right)$ by Lemma 2 , we may deduce that

$$
\begin{aligned}
& (t-1)(t-2) F(G, t)=(3-t)\left[(3-t) F\left(G_{1}, t\right) F\left(G_{2}, t\right)\right. \\
& \left.\quad-(t-2) F\left(G_{1}, t\right) F\left(G_{2}-x y, t\right)-(t-2) F\left(G_{1}-x y, t\right) F\left(G_{2}, t\right)\right] .
\end{aligned}
$$

Let $r$ be a real number such that $3-t-2 r \geq 0$. Then

$$
\begin{aligned}
& (t-1)(t-2) F(G, t)=(3-t)\left[(3-t-2 r) F\left(G_{1}, t\right) F\left(G_{2}, t\right)+\right. \\
& \quad+F\left(G_{1}, t\right)\left(r F\left(G_{2}, t\right)-(t-2) F\left(G_{2}-x y, t\right)\right)+ \\
& \left.\quad+F\left(G_{2}, t\right)\left(r F\left(G_{1}, t\right)-(t-2) F\left(G_{1}-x y, t\right)\right)\right] .
\end{aligned}
$$

Since $t \in(2,3)$ and $3-t-2 r \geq 0$, we may apply the inductive hypothesis to $G_{1}, G_{2}$ to obtain

$$
\begin{aligned}
& (t-1)(t-2) F(G, t) \geq(3-t) \times \\
& \quad\left[(3-t-2 r) F\left(G_{1}, t\right) F\left(G_{2}, t\right)+2 g(r g-t+2) F\left(G_{1}-x y, t\right) F\left(G_{2}-x y, t\right)\right] .
\end{aligned}
$$

In order to compare $F(G, t)$ and $F(G-x y, t)$, we choose a value for $r$ so that the ratio of the coefficients in (2) and (3) are equal. Let $r=\frac{1}{2}(t-2)(3-t+2 g)\left(g^{2}+\right.$ $t-2)^{-1}$. Then $3-t-2 r=g\left(g^{2}+t-2\right)^{-1} f(t, g) \geq 0$ by the hypotheses of the lemma. Thus we may use (2) and (3) to obtain

$$
F(G, t) \geq\left[\frac{(3-t)^{2}}{t-2}-\frac{(3-t)(3-t+2 g)}{g^{2}+t-2}\right] F(G-x y, t) .
$$

Since $h(t, g) \geq 0$ we have $F(G, t) \geq g F(G, t)$ and (b) holds for $G$. The truth of (c) now follows from (a) and (b).

We next choose a value for $g$ in Lemma 17 in order to maximise the range of values of $t$ for which the lemma can be applied. It can be seen that the maximum 
value of $t \in(2,3)$ for which the contraint $h(t, g) \geq 0$ holds occurs when $h(t, g)=$ $0=\frac{\partial h}{\partial g}$. Solving these simultaneous equations we obtain $t=\alpha$ and $g=\rho$ where $\rho \approx 1.33 \ldots$ is the zero in $(1,2)$ of the polynomial $g^{4}+2 g^{3}-2 g^{2}-4 g+1$. Since $f(t, \rho)$ and $h(t, \rho)$ are decreasing functions of $t$ for $t \in(2, \alpha)$ and $f(\alpha, \rho)>0=$ $h(\alpha, \rho)$, we have:

Corollary 18 Let $(G, x) \in \mathcal{L}(x, y)$ and $t \in(2, \alpha]$. Then:

(a) $F(G-x y, t) \geq 0$ with strict inequality when $G \neq L_{0}$;

(b) $F(G, t) \geq \rho F(G-x y, t)$, where $\rho \approx 1.33 \ldots$ is the zero in $(1,2)$ of the polynomial $g^{4}+2 g^{3}-2 g^{2}-4 g+1$;

(c) $F(G, t)>0$

We now use this Corollary to prove that no extension of $\left(K_{4}, x\right)$ can have a flow root in $(2, \alpha]$.

Lemma 19 Let $(G, x)$ be an extension of $\left(K_{4}, x\right)$. Then $F(G, t)<0$ for all $t \in(2, \alpha]$.

Proof. Let $e_{1}, e_{2}, e_{3}$ be the edges of $\left(K_{4}, x\right)$ which are not incident to $x$. Since $(G, x)$ is an extension of $\left(K_{4}, x\right)$, there exist graphs $\left(G_{1}, x\right),\left(G_{2}, x\right)$, and $\left(G_{3}, x\right)$ in $\mathcal{L}(x, y)$ such that $G-\left\{e_{1}, e_{2}, e_{3}\right\}=\left(G_{1}-y\right) \cup\left(G_{2}-y\right) \cup\left(G_{3}-y\right),\left(G_{i}-y\right) \cap\left(G_{i+1}-y\right)=$ $\{x\}$, and $e_{i}$ is incident with a vertex of $G_{i}-x$ and $G_{i+1}-x$ for all $1 \leq i \leq 3$, reading subscripts modulo three. Applying Lemmas 8,7, 6 and 2 to $G$ we may deduce that

$$
\begin{aligned}
(t-1)^{2}(t-2)^{2} F(G, t)= \\
\quad(t-3) F\left(G_{1}, t\right) F\left(G_{2}, t\right) F\left(G_{3}, t\right)+(t-2) F\left(G_{1}, t\right) F\left(G_{2}, t\right) F\left(G_{3}-x y, t\right)+ \\
\quad(t-2) F\left(G_{1}, t\right) F\left(G_{2}-x y, t\right) F\left(G_{3}, t\right)+(t-2) F\left(G_{1}-x y, t\right) F\left(G_{2}, t\right) F\left(G_{3}, t\right) \\
\quad+(t-2)^{2} F\left(G_{1}-x y, t\right) F\left(G_{2}-x y, t\right) F\left(G_{3}-x y, t\right) .
\end{aligned}
$$

If $G_{1}=G_{2}=G_{3}=L_{0}$ then $G=K_{4}, F(G, t)=(t-1)(t-2)(t-3)$ and the lemma holds for $G$. Hence we may suppose that $G_{1} \neq L_{0}$.

Suppose that $G_{2}=G_{3}=L_{0}$. Then $F\left(G_{i}, t\right)=(t-1)(t-2)$ and $F\left(G_{i}-x y, t\right) \equiv 0$ for all $2 \leq i \leq 3$. Substituting into (4), we obtain

$$
F(G, t)=(t-3) F\left(G_{1}, t\right)+(t-2) F\left(G_{1}-x y, t\right) .
$$

Corollary 18 and the fact that $t \in(2, \alpha]$ now give

$$
F(G, t) \leq-[\rho(3-\alpha)-(\alpha-2)] F\left(G_{1}-x y, t\right)<0 .
$$


Hence we may suppose that $G_{1}, G_{2} \neq L_{0}$.

Suppose that $G_{3}=L_{0}$. Then $F\left(G_{3}\right)=(t-1)(t-2)$ and $F\left(G_{3}-x y\right) \equiv 0$. Substituting into (4), we obtain

$$
\begin{aligned}
(t-1) & (t-2) F(G, t) \\
= & (t-3) F\left(G_{1}, t\right) F\left(G_{2}, t\right)+ \\
& (t-2)\left[F\left(G_{1}-x y, t\right) F\left(G_{2}, t\right)+F\left(G_{1}, t\right) F\left(G_{2}-x y, t\right)\right] \\
= & -\frac{1}{2} F\left(G_{1}, t\right)\left[(3-t) F\left(G_{2}, t\right)-2(t-2) F\left(G_{2}-x y, t\right)\right]- \\
& \frac{1}{2} F\left(G_{2}, t\right)\left[(3-t) F\left(G_{1}, t\right)-2(t-2) F\left(G_{1}-x y, t\right)\right] .
\end{aligned}
$$

Corollary 18 and the fact that $t \in(2, \alpha]$ now give

$$
(t-1)(t-2) F(G, t) \leq-\rho[\rho(3-\alpha)-2(\alpha-2)] F\left(G_{1}-x y, t\right) F\left(G_{2}-x y, t\right)<0 .
$$

Hence we may suppose that $G_{1}, G_{2}, G_{3} \neq L_{0}$.

We may rewrite (4) as

$$
\begin{aligned}
& (t-1)^{2}(t-2)^{2} F(G, t)= \\
& \frac{1}{3} F\left(G_{1}, t\right) F\left(G_{2}, t\right)\left[(3-t) F\left(G_{3}, t\right)-3(t-2) F\left(G_{3}-x y, t\right)\right]- \\
& \frac{1}{3} F\left(G_{1}, t\right) F\left(G_{3}, t\right)\left[(3-t) F\left(G_{2}, t\right)-3(t-2) F\left(G_{2}-x y, t\right)\right]- \\
& \frac{1}{3} F\left(G_{2}, t\right) F\left(G_{3}, t\right)\left[(3-t) F\left(G_{1}, t\right)-3(t-2) F\left(G_{1}-x y, t\right)\right]+ \\
& \quad(t-2)^{2} F\left(G_{1}-x y, t\right) F\left(G_{2}-x y, t\right) F\left(G_{3}-x y, t\right) .
\end{aligned}
$$

Corollary 18 and the fact that $t \in(2, \alpha]$ now give

$$
\begin{gathered}
(t-1)^{2}(t-2)^{2} F(G, t) \leq-\left[(3-\alpha) \rho^{3}-3(\alpha-2) \rho^{2}-(\alpha-2)^{2}\right] \times \\
F\left(G_{1}-x y, t\right) F\left(G_{2}-x y, t\right) F\left(G_{3}-x y, t\right)<0 .
\end{gathered}
$$

Our final result in this subsection is to show that there exists a sequence of extensions of $\left(K_{4}, x\right)$ with flow roots converging to $\alpha$ from above. Let $\left(G_{1}, x\right)$ be a copy of $\left(K_{4}, x\right)$ and $e$ be an edge of $G_{1}$ incident to $x$. For $i \geq 2$, let $\left(G_{i}, x\right)$ be the near-cubic graph obtained from $\left(G_{i-1}, x\right)$ by applying the single edge extension operation to every edge incident to $x$ other than $e$.

Theorem 20 For all $\varepsilon>0$ there exists an $n \geq 1$ such that $G_{n}$ has a flow root in $(\alpha, \alpha+\varepsilon]$. 
Proof. Let $\left(L_{0}, x\right)$ be the near-cubic graph defined in the paragraph before Lemma 17. For $i \geq 1$, let $\left(L_{i}, x\right)$ be the near cubic graph obtained from $\left(L_{i-1}, x\right)$ by applying the single edge extension operation to every edge incident to $x$ other than $x y$. For $i \geq 0$, let $a_{i}(t)=F\left(L_{i}, t\right), b_{i}(t)=F\left(L_{i}-x y, t\right)$, and $c_{i+1}(t)=F\left(G_{i+1}, t\right)$. Applying the argument used in the derivation of (2), we deduce that for $i \geq 0$,

$$
(t-1) b_{i+1}=a_{i}^{2}+(t-2) b_{i}^{2} .
$$

Applying the argument used in the derivation of (5), we deduce that for $i \geq 0$,

$$
(t-1)(t-2) c_{i+1}=(t-3) a_{i}^{2}+2(t-2) a_{i} b_{i} .
$$

Applying Lemma 7 to $L_{i}$ for $i \geq 1$, using the 3-edge-cut incident to the triangle containing $y$, we have

$$
a_{i}=(t-3) c_{i} .
$$

For $i \geq 1$, let $r_{i}(t)=c_{i}(t) / b_{i}(t)$. Thus $r_{1}(t)=(t-3) /(t-2)$ and equations (6), (7) and (8) imply that

$$
r_{i+1}(t)=\frac{(t-3)\left[(t-3)^{2} r_{i}^{2}+2(t-2) r_{i}\right]}{(t-2)\left[(t-3)^{2} r_{i}^{2}+(t-2)\right]} .
$$

Let

$$
f(r, t)=\frac{(t-3)\left[(t-3)^{2} r^{2}+2(t-2) r\right]}{(t-2)\left[(t-3)^{2} r^{2}+(t-2)\right]} .
$$

Thus $r_{i+1}(t)=f\left(r_{i}(t), t\right)$ for all $i \geq 1$.

Claim 2 If $r<0$ and $t \in(\alpha, 3)$ then $f(r, t)>r$.

Proof. We have

$$
f(r, t)-r=-r(t-2)^{-1}\left[(3-t)^{2} r^{2}+(t-2)\right]^{-1} g(r, t),
$$

where

$$
g(r, t)=(3-t)\left[(3-t)^{2} r+2(t-2)\right]+(t-2)\left[(3-t)^{2} r^{2}+(t-2)\right] .
$$

Solving $g(r, t)=0$ for $r$, we obtain

$$
r=\frac{t^{2}-6 t+9 \pm \sqrt{t^{4}-8 t^{3}+22 t^{2}-28 t+17}}{2(t-2)(t-3)} .
$$

Since $t^{4}-8 t^{3}+22 t^{2}-28 t+17<0$ for $t \in(\alpha, 3)$ we deduce that $g(r, t)>0$ for all $r$ and all $t \in(\alpha, 3)$. Thus $f(r, t)-r>0$ for all $r<0$ and all $t \in(\alpha, 3)$. 
Claim 3 If $t \in(\alpha, 3)$ then $r_{n}(t) \geq 0$ for some $n \geq 1$.

Proof. Suppose $r_{n}(t)<0$ for all $n \geq 1$. Since $r_{i+1}(t)=f\left(r_{i}(t), t\right)$, Claim 2 imples that $\left\{r_{i}(t): i \geq 1\right\}$ is a monotonicly increasing sequence bounded above by zero. Hence it must converge to a limit $r \leq 0$ satisfying $r=f(r, t)$. Using Claim 2, we may deduce that $r=0$. This is impossible since for fixed $t \in(\alpha, 3)$, the right hand side of (9) is positive when $r_{i}(t)$ is negative and sufficiently close to zero.

We may now complete the proof of the theorem. By Claim 3, we may choose $n \geq 1$ such that $r_{n}(\alpha+\varepsilon) \geq 0$. Since (6) implies that $b_{n}(\alpha+\varepsilon) \geq 0$, we must have $c_{n}(\alpha+\varepsilon) \geq 0$. On the other hand, Lemma 19 implies that $c_{n}(\alpha)<0$. Since $F\left(G_{n}, t\right)=c_{n}(t)$ is continuous, it must have a zero in $(\alpha, \alpha+\varepsilon]$.

\subsubsection{The zero-free interval}

We show that no near-cubic graph can have a flow root in $(2, \alpha]$. We shall need the following 'characterisation' of the family of near-cubic graphs which are extensions of $\left(K_{4}, x\right)$. We only state and prove the direction of the characterization which we require. The reader may find it helps their understanding of the following proof if they first verify that the graph in Figure 3 satisfies conditions (a) and (b) below.

Lemma 21 Let $(G, x)$ be a 3-connected near-cubic graph and $T$ be the cleavage unit tree of $G-x$. Suppose that:

(a) each non-circuit cleavage unit of $G-x$ is a $K_{2}^{3}$ and has degree two in $T$;

(b) each circuit cleavage unit of $G-x$ either is a $C_{4}$ and has degree one in $T$, or is a $C_{5}$ and has degree two in $T$, or is a $C_{6}$ and has degree three in $T$.

Then $(G, x)$ is an extension of $\left(K_{4}, x\right)$.

Proof. Let $P=X_{1} X_{2} \ldots X_{r}$ be a longest path in $T$. Then $X_{1}$ has degree one in $T$ so is a $C_{4}$-cleavage unit of $G-x$ by (b). Let $X_{1}=u_{1} u_{2} u_{3} u_{4} u_{1}$ where $u_{1}, u_{2}$ have degree two in $G-x$. Since $G$ is 3-connected, $x u_{1}, x u_{2} \in E(G)$. By Lemma 12(a) and hypothesis (a) of the lemma, $X_{2}$ is a $K_{2}^{3}$-cleavage unit of $G-x$ and has degree two in $T$. Thus $V\left(X_{2}\right)=\left\{u_{3}, u_{4}\right\}$ and $u_{3} u_{4} \in E(G)$. By Lemma 12(a), $X_{3}$ is a circuit cleavage unit of $G$. By (b), $X_{3}$ has degree at most three in $T$. If $X_{3}$ has degree one in $T$ then, by hypothesis (b), $X_{3}$ is a $C_{4}$-cleavage unit of $G-x$ and $(G, x)$ is a single edge extension of $\left(K_{4}, x\right)$. Thus we may assume that $X_{3}$ has degree two or three in $T$. 
Suppose $X_{3}$ has degree two in $T$. By (b), $X_{3}$ is a $C_{5}$-cleavage unit of $G-x$. Let $X_{3}=u_{3} v z w u_{4} u_{3}$. By Lemma 16 and symmetry, we may suppose that the virtual edge of $G-x$ contained in $X_{3}$ and distinct from $u_{3} u_{4}$, is $w z$. Thus $v$ is a vertex of degree two in $G-x$ and $x v \in E(G)$. Let $\left(G^{\prime}, x\right)$ be the near-cubic graph obtained from $G-\left\{u_{1}, u_{2}, u_{3}, u_{4}\right\}$ by adding a new vertex $u$ and edges $u x, u v, u w$. Then $(G, x)$ is a single edge extension of $\left(G^{\prime}, x\right)$. Furthermore $G^{\prime}$ is 3 -connected, the set of cleavage units of $G^{\prime}$ is obtained from the set of cleavage units of $G$ by deleting $X_{1}, X_{2}, X_{3}$ and adding a new $C_{4}$-cleavage unit $X^{\prime}=u v z w u$, and the cleavage unit tree $T^{\prime}$ of $G^{\prime}-x$ is obtained from $T-\left\{X_{1}, X_{2}, X_{3}\right\}$ by adding the new vertex $X^{\prime}$ and edge $X^{\prime} X_{4}$. Thus $G^{\prime}$ satisfies the hypotheses of the Lemma. By induction, $\left(G^{\prime}, x\right)$ is an extension of $\left(K_{4}, x\right)$. Thus $(G, x)$ is an extension of $\left(K_{4}, x\right)$.

Suppose $X_{3}$ has degree three in $T$. By (b), $X_{3}$ is a $C_{6}$-cleavage unit of $G-x$. Let $X_{3}=u_{3} v y z w u_{4} u_{3}$. By Lemma 16, the virtual edges of $G-x$ contained in $X_{3}$ are $u_{3} u_{4}, w y, z w$. By symmetry, we may assume that $v y \in E\left(X_{4}\right)$. Let $Y_{2}$ be the neighbour of $X_{3}$ in $T$ distinct from $X_{4}$. As above, $Y_{2}$ is a $K_{2}^{3}$-cleavage unit of $G-x$ and has degree two in $T$. Thus $V\left(Y_{2}\right)=\{w, z\}$ and $w z \in E(G)$. Let $Y_{1}$ be the neighbour of $Y_{2}$ in $T$ distinct from $X_{3}$. The maximality of $P$ implies that $Y_{1}$ is an end-vertex of $T$ and hence is a $C_{4}$-cleavage unit of $G$. Let $\left(G^{\prime}, x\right)$ be the nearcubic graph obtained from $G-\left\{u_{1}, u_{2}, u_{3}, u_{4}\right\}$ by adding a new vertex $u$ and edges $u x, u v, u w$. Then $(G, x)$ is a single edge extension of $\left(G^{\prime}, x\right)$. Furthermore $G^{\prime}$ is 3connected, the set of cleavage units of $G^{\prime}$ is obtained from the set of cleavage units of $G$ by deleting $X_{1}, X_{2}, X_{3}$ and adding a new $C_{5}$-cleavage unit $X^{\prime}=w u v y z w$, and the cleavage unit tree $T^{\prime}$ of $G^{\prime}-x$ is obtained from $T-\left\{X_{1}, X_{2}, X_{3}\right\}$ by adding the new vertex $X^{\prime}$ and edges $X^{\prime} X_{4}, X^{\prime} Y_{2}$. Thus $G^{\prime}$ satisfies the hypotheses of the Lemma. By induction, $\left(G^{\prime}, x\right)$ is an extension of $\left(K_{4}, x\right)$. Thus $(G, x)$ is an extension of $\left(K_{4}, x\right)$.

Let $C_{k}$ denote the isomorphism class of circuits of length $k$.

Theorem 22 Let $(G, x)$ be a non-separable near-cubic graph with $m$ edges, $n$ vertices and $c$ non-circuit cleavage units. Then $F(G, t)$ is non-zero with sign $(-1)^{m-n+c}$ for $t \in(2, \alpha]$, where $\alpha \approx 2.225 \ldots$ is the real zero in $(2,3)$ of the polynomial $t^{4}-8 t^{3}+22 t^{2}-28 t+17$.

Proof. We proceed by contradiction. Suppose the theorem is false. Then we may choose a $t \in(2, \alpha]$ and a near-cubic graph $(G, x)$ satisfying the hypotheses of the theorem such that $(-1)^{m-n+c} F(G, t) \leq 0$ and such that, for this fixed value of $t, m$ is as small as possible. We shall show that $G$ satisfies the hypotheses of Lemma 21 and hence is an extension of $\left(K_{4}, x\right)$. This will give the required contradiction by Lemma 19. Since the theorem holds if $G$ is a circuit or a 3-bond, we deduce that $G$ is 2 -connected, $n \geq 3$ and $m \geq 4$. Since suppressing vertices of degree two in $G$ 
leaves $m-n$ and $c$ unchanged and also leaves $F(G, t)$ unchanged by Lemma 3, $G$ has minimum degree three.

\section{Claim 4 G is 3-connected.}

Proof. Suppose the claim is false and let $G_{1}$ be an end-cleavage unit of $G$. Since $G$ has minimum degree three, $G_{1}$ is not a circuit, and hence $G_{1}$ is either a 3-bond or is 3-connected. We may express $G$ as $G=H_{1} \cup H_{2}$ with $H_{1} \cap H_{2}=\{u, v\}$ for some $u, v \in V(G)$ and $G_{1}=H_{1}+u v$. Without loss of generality $x \neq v$. Let $G_{2}=H_{2}+u v$. Since $G$ is near-cubic and $d_{G_{1}}(v)=3$, we have $d_{G_{2}}(v)=2$. For $i=1,2$, let $x_{i}=x$ if $x \in V\left(H_{i}\right)$ and otherwise relabel an arbitrary vertex of $G_{i}$ as $x_{i}$. Then $\left(G_{i}, x_{i}\right)$ is near-cubic and non-separable, $m=m_{1}+m_{2}-2, n=n_{1}+n_{2}-2, c_{1}=1$ and $c_{2}=c-1$. By Lemma 5, $(t-1) F(G, t)=F\left(G_{1}, t\right) F\left(G_{2}, t\right)$. Since $m_{1}, m_{2} \geq 3$, we have $m_{1}, m_{2} \leq m-1$. Thus we may apply induction to $G_{1}$ and $G_{2}$ to deduce that the theorem holds for $G$.

\section{Claim 5 G is cyclically 4-edge-connected.}

Proof. Suppose the claim is false. Let $S$ be a cyclic 3-edge-cut in $G$ and $H_{1}, H_{2}$ be the components of $G-S$. For $i=1,2$, let $G_{i}$ be the near-cubic graph obtained from $G$ by contracting $E\left(H_{3-i}\right)$. Since $G$ is 3-connected, $G_{1}$ and $G_{2}$ are 3-connected, and hence $c=1=c_{1}=c_{2}$. Furthermore $m=m_{1}+m_{2}+3, n=n_{1}+n_{2}+2$ and, by Lemma 7, $(t-1)(t-2) F(G, t)=F\left(G_{1}, t\right) F\left(G_{2}, t\right)$. Applying induction to $G_{1}$ and $G_{2}$ we deduce that the theorem holds for $G$.

Using Claim 5 we immediately deduce:

Claim $6 G-e$ is essentially 3-connected for all $e \in E(G)$.

Claim $7 \mathrm{G} / \mathrm{e}$ is a cleavage unit path with an even number of non-circuit cleavage units for all edges e of $G$ incident to $x$.

Proof. Let $G_{1}=G / e$ and $G_{2}=G-e$. By Claim $4, G_{1}$ is 2-connected and by Claim $6, G_{2}$ is essentially 3-connected. By Lemma $2, F(G, t)=F\left(G_{1}, t\right)-F\left(G_{2}, t\right)$. Since $n=n_{1}+1=n_{2}, m=m_{1}-1=m_{2}-1$ and $c=1=c_{2}$, we may deduce that the theorem will hold for $G$ by applying induction to $G_{1}$ and $G_{2}$ if $c_{1}$ is odd. Hence $c_{1}$ is even.

Let $e=x v$ and let $x_{1}$ be the vertex of $G_{1}$ obtained by contracting $e$. Choose an end cleavage unit $H_{1}$ of $G_{1}$. Then we may express $G_{1}$ as $G_{1}=\left(H_{1}-y z\right) \cup\left(H_{2}-\right.$ $y z$ ) where $H_{1} \cap H_{2}=\{y, z, y z\}$ for some $y, z \in V\left(G_{1}\right)$. Since $G$ is 3-connected by Claim $4, x_{1} \in\{y, z\}$, say $x_{1}=y$. Then $G-\{x, v, z\}$ is disconnected. Since $G$ is 
3-connected, some vertex of $V\left(H_{1}\right)-\left\{x_{1}, z\right\}$ is adjacent to $v$ in $G$. Thus every end cleavage unit of $G_{1}$ has an internal vertex which is a neighbour of $v$ in $G$ distinct from $x$. Since $G$ is near-cubic, $v$ has exactly two neighbours distinct from $x$. Thus $G_{1}$ has exactly two end cleavage units and hence $G_{1}$ is a cleavage unit path.

Claim 8 Suppose $S=\left\{u_{1} u_{2}, v_{1} v_{2}\right\}$ is a 2-edge-cut of $G-x$. Let $G-S=H_{1} \cup H_{2}$ where $H_{1} \cap H_{2}=\{x\}, u_{1}, v_{1} \in V\left(H_{1}\right)$ and $u_{2}, v_{2} \in V\left(H_{2}\right)$. Suppose further that $d_{H_{1}}(x) \geq 2$ and $d_{H_{2}}(x) \geq 2$. Let $G^{-}$be the graph obtained from $G-x$ by adding two new vertices $x_{1}, x_{2}$, and joining $x_{i}$ to each neighbour of $x$ in $H_{i}$ for $1 \leq i \leq 2$. Let $G^{+}$be obtained from $G^{-}$by adding a new edge $e=x_{1} x_{2}$. For $i=1,2$, let $G_{i}^{+}$ be the graph obtained from $\mathrm{G}^{+}$by contracting $E\left(\mathrm{H}_{3-i}\right)$ to the single vertex $y_{i}$, and $G_{i}^{-}=G_{i}^{+}-x_{i} y_{i}$. Then:

(a) $G^{-}$is a cleavage unit path and $c^{-}=c_{1}^{-}+c_{2}^{-}$is odd;

(b) if $x$ is adjacent to a vertex in $\left\{u_{i}, v_{i}\right\}$ for some $i \in\{1,2\}$, then $G_{i}^{-}$is essentially 3-connected;

(c) $x$ is not adjacent to both a vertex in $\left\{u_{1}, v_{1}\right\}$ and a vertex in $\left\{u_{2}, v_{2}\right\}$;

(d) if $c_{2}^{-}$is odd, then $c\left(G_{2}^{+} / x_{2} y_{2}\right)$ is even and $c_{2}^{-}=1$.

\section{Proof.}

(a) The fact that $G^{-}$is a cleavage unit path follows since the 3-connectivity of $G$ implies that each end cleavage unit of $G^{-}$must contain either $x_{1}$ or $x_{2}$ as an internal vertex. By Lemma 8,

$$
(t-1)(t-2) F(G, t)=F\left(G_{1}^{+}, t\right) F\left(G_{2}^{+}, t\right)+(t-2) F\left(G_{1}^{-}, t\right) F\left(G_{2}^{-}, t\right) .
$$

Since $G$ is 3-connected, $G_{1}^{+}$and $G_{2}^{+}$are both 3-connected. Thus $c_{1}^{+}=1=c_{2}^{+}$. Furthermore

$$
m=m_{1}^{+}+m_{2}^{+}-4=m_{1}^{-}+m_{2}^{-}-2
$$

and

$$
n=n_{1}^{+}+n_{2}^{+}-3=n_{1}^{-}+n_{2}^{-}-3 .
$$

If $c_{1}^{-}+c_{2}^{-}$is even, then we may deduce that the theorem holds for $G$ from (10) by applying induction to $G_{1}^{+}, G_{2}^{+}, G_{1}^{-}, G_{2}^{-}$. Thus $c^{-}=c_{1}^{-}+c_{2}^{-}$is odd.

(b) Suppose $x$ is adjacent to $u_{1}$. Since $G_{1}^{+}$is 3 -connected and $G_{1}^{+} / x_{1} y_{1}$ is not 3 -connected $\left(u_{1}\right.$ has only two neighbours in $\left.G_{1}^{+} / x_{1} y_{1}\right)$, Lemma 10 implies that $G_{1}^{-}=G_{1}^{+}-x_{1} y_{1}$ is essentially 3 -connected.

(c) Suppose $x$ is adjacent to both a vertex in $\left\{u_{1}, v_{1}\right\}$ and a vertex in $\left\{u_{2}, v_{2}\right\}$. Using 
(b) we deduce that $G_{1}^{-}$and $G_{2}^{-}$are both essentially 3-connected. Thus $c_{1}^{-}=c_{2}^{-}=1$. This is impossible since $c_{1}^{-}+c_{2}^{-}=c^{-}$is odd by (a).

(d) Henceforth we assume that $c_{2}^{-}$is odd. Then $c_{1}^{-}$is even by (a). If $\left|V\left(H_{2}\right)\right|=3$ then $G_{2}^{+} \cong K_{4}$ and (d) holds. Thus we may assume that $\left|V\left(H_{2}\right)\right| \geq 4$.

Let $G_{2}=G_{2}^{+} / x_{2} y_{2}$, and suppose that $c_{2}$ is odd. By Lemma $2, F\left(G_{2}^{+}, t\right)=$ $F\left(G_{2}, t\right)-F\left(G_{2}^{-}, t\right)$. Substitution into (10) gives

$$
\begin{aligned}
(t-1)(t-2) F(G, t)= & F\left(G_{1}^{+}, t\right) F\left(G_{2}, t\right)- \\
& {\left[F\left(G_{1}^{+}, t\right)-(t-2) F\left(G_{1}^{-}, t\right)\right] F\left(G_{2}^{-}, t\right) . }
\end{aligned}
$$

Let $G_{1}^{*}$ be the graph obtained from $G_{1}^{+}-y_{1}$ by adding three new vertices $z_{1}, z_{2}, z_{3}$ and edges $z_{1} x_{1}, z_{2} u_{1}, z_{3} v_{1}, z_{1} z_{2}, z_{2} z_{3}, z_{3} z_{1}$, and let $G_{1}^{\prime}=G_{1}^{*} / x_{1} z_{1}$. Since $G_{1}^{+}$ is 3-connected, $G_{1}^{*}$ and $G_{1}^{\prime}$ are 3-connected and $c_{1}^{\prime}=1$. Applying induction to $G_{1}^{\prime}$, we deduce that $(-1)^{m_{1}^{\prime}-n_{1}^{\prime}+1} F\left(G_{1}^{\prime}, t\right)>0$. Applying Lemma 2 to $G_{1}^{*}$ using the edge $x_{1} z_{1}$, and then using Lemmas 5 and 7 , we deduce that

$$
F\left(G_{1}^{\prime}, t\right)=(t-3) F\left(G_{1}^{+}, t\right)+(t-2) F\left(G_{1}^{-}, t\right) .
$$

Thus $(-1)^{m_{1}^{\prime}-n_{1}^{\prime}+1}\left[(t-3) F\left(G_{1}^{+}, t\right)+(t-2) F\left(G_{1}^{-}, t\right)\right]>0$. Since $m_{1}^{\prime}=m_{1}^{+}+2$ and $n_{1}^{\prime}=n_{1}^{+}+1$ we have

$$
(3-t)(-1)^{m_{1}^{+}-n_{1}^{+}+1} F\left(G_{1}^{+}, t\right)-(t-2)(-1)^{m_{1}^{+}-n_{1}^{+}+1} F\left(G_{1}^{-}, t\right)>0 .
$$

Since $0<3-t<1$, and $(-1)^{m_{1}^{+}-n_{1}^{+}+1} F\left(G_{1}^{+}, t\right)>0$ by induction, we have

$$
(-1)^{m_{1}^{+}-n_{1}^{+}+1}\left[F\left(G_{1}^{+}, t\right)-(t-2) F\left(G_{1}^{-}, t\right)\right]>0 .
$$

Thus $F\left(G_{1}^{+}, t\right)-(t-2) F\left(G_{1}^{-}, t\right)$ is non-zero with sign $(-1)^{m_{1}^{+}-n_{1}^{+}+1}$. Substituting into (11), applying induction to $G_{1}^{+}, G_{2}, G_{2}^{-}$, and using $m=m_{1}^{+}+m_{2}-3=$ $m_{1}^{+}+m_{2}^{-}-3, n=n_{1}^{+}+n_{2}-2=n_{1}^{+}+n_{2}^{-}-3, c=1=c_{1}^{+}$and $c_{2}, c_{2}^{-}$are odd, we deduce that $F(G, t)$ is non-zero with sign $(-1)^{m-n+1}$. This contradicts the choice of $G$. Thus the assumption that $c_{2}$ is odd must be false. Hence $c_{2}$ is even and, in particular, $G_{2}$ is not 3-connected. Lemma 10 now implies that $G_{2}^{-}=G_{2}^{+}-x_{2} y_{2}$ is essentially 3 -connected and hence $c_{2}^{-}=1$.

Claim 9 Let $X$ be an end cleavage unit of $G-x$. Then $X \cong C_{4}$.

Proof. Since $G$ is 3-connected, $x$ is adjacent to at least one internal vertex $v$ of $X$. Thus $d_{G-x}(v)=2=d_{X}(v)$. Hence $X$ is neither 3-connected nor a 3-bond, so $X \cong C_{r}$ for some $r \geq 3$. Let $X=v_{1} v_{2} \ldots v_{r} v_{1}$, where $v_{i}$ is an internal vertex of $X$ for 
$2 \leq i \leq r-1$. Then, for $2 \leq i \leq r-1$, we have $d_{X}\left(v_{i}\right)=2=d_{G-x}\left(v_{i}\right)$ and hence $e_{i}=x v_{i} \in E(G)$. Since $d_{G-x}\left(v_{1}\right), d_{G-x}\left(v_{r}\right) \geq 3$, we have $x v_{1}, x v_{r} \notin E(G)$. If $r=3$, then $G-\left\{x, v_{2}\right\}$ is 2-connected and Lemma 13 implies that $G / e_{2}$ is 3 -connected. This would contradict Claim 7 and hence $r \geq 4$.

Let $S=\left\{v_{1} v_{2}, v_{3} v_{4}\right\}$. Then $S$ is a 2-edge-cut of $G-x$. We have $G-S=H_{1} \cup H_{2}$ where $H_{1} \cap H_{2}=\{x\}$ and $V\left(H_{1}\right)=\left\{x, v_{2}, v_{3}\right\}$. Let $G^{-}, G_{1}^{-}, G_{2}^{-}$be as defined in the statement of Claim 8. Then $G_{1}^{-}$is homeomorphic to $K_{2}^{3}$ so $c_{1}^{-}=1$.

Suppose $r \geq 5$. Then $x v_{4} \in E(G)$ so by Claim 8(b), $G_{2}^{-}$is essentially 3connected. Thus $c_{2}^{-}=1$ and $c^{-}=c_{1}^{-}+c_{2}^{-}=2$. This contradicts Claim 8(a) and hence $r=4$.

Claim 10 Let $X$ be a non-circuit cleavage unit of $G-x$ and $u_{2} v_{2}$ be a virtual edge of $G-x$ contained in $X$. Let $S=\left\{u_{1} u_{2}, v_{1} v_{2}\right\}$ be the 2-edge-cut of $G-x$ incident to $\left\{u_{2}, v_{2}\right\}$. Let $G^{-}, G_{1}^{+}, G_{2}^{+}, G_{1}^{-}, G_{2}^{-}$be as defined in the statement of Claim 8. Then:

(a) $c_{2}^{-}$is even and $c_{1}^{-}=1$;

(b) $X$ has degree two in the cleavage unit tree $T$ of $G-x$;

Proof. Note that Claim 9 implies that $d_{G_{1}^{-}}(x), d_{G_{2}^{-}}(x) \geq 2$.

(a) Suppose $c_{2}^{-}$is odd. Then Claim 8(d) implies that $G_{2}=G_{2}^{+} / x_{2} y_{2}$ is not 3connected. Since $G_{2}^{+}$is 3-connected and near-cubic, we may use Lemma 13 to deduce that $G_{2}^{+}-\left\{x_{2}, y_{2}\right\}$ has a cut edge $f$. Thus $u_{2}$ and $v_{2}$ belong to different components of $G_{2}^{-}-\left\{x_{2}, y_{2}, f\right\}$. This contradicts the fact that $u_{2}$ and $v_{2}$ belong to the same non-circuit cleavage unit $X$ of $G-x$ and hence are joined by three internally disjoint paths in $G-x$. Thus $c_{2}^{-}$is even, and by Claim $8(\mathrm{a}),(\mathrm{d}), c_{1}^{-}=1$.

(b) Suppose $X$ had degree at least three in $T$. Let $e^{*}$ be the edge of $T$ corresponding to the virtual edge $u_{2} v_{2}$ of $G-x$, and $T_{2}$ be the component of $T-e^{*}$ which contains $X$. Then $T_{2}$ is the cleavage unit tree of the graph $G_{2}^{*}$ obtained by suppressing $y_{2}$ in $G_{2}^{-}$. Since $X$ has degree at least three in $T$, it has degree at least two in $T_{2}$. Thus $X$ is not an end cleavage unit of $G_{2}^{*}$. Since $G$ is 3-connected, $x$ is adjacent to an internal vertex in each end cleavage unit of $G-x$. Thus $x_{2}$ is adjacent to an internal vertex in each end cleavage unit of $G_{2}^{*}-x_{2}$. By Lemma 12(b), $G_{2}^{*}$ is essentially 3 -connected. Since $G_{2}^{-}$is homeomorphic to $G_{2}^{*}, G_{2}^{-}$is essentially 3-connected. Hence $c_{2}^{-}=1$. This contradicts (a) and completes the proof of (b).

Claim 11 Let $X$ be a 3-connected cleavage unit of $G-x$ and $u v, w z$ be the virtual edges of $G-x$ contained in $X$. Let $X^{*}$ be obtained from $X-\{u v, w z\}$ by adding a new vertex $y$ and new edges $y u, y v, y w, y z$. Then $X^{*}$ is 3-connected. 
Proof. Since $X$ is 3-connected, no vertex of $X$ is adjacent to $x$ in $G$. Suppose $X^{*}$ is not 3-connected. Then $y$ is contained in a 2-vertex-cut of $X^{*}$. Since each vertex of $X^{*}$ other than $y$ has degree three, this implies that $X^{*}-\{y, e\}$ is disconnected for some $e \in E\left(X^{*}\right)$. Thus $S=\{u v, w z, e\}$ is a 3-edge-cut of $X$. Let $X_{1}$ and $X_{2}$ be the components of $X-S$. Relabelling if necessary we may suppose that $u, w \in V\left(X_{1}\right)$ and $v, z \in V\left(X_{2}\right)$. Let $S_{1}=\left\{u u_{1}, v v_{1}\right\}$ and $S_{2}=\left\{w w_{1}, z z_{1}\right\}$ be the 2-edge-cuts of $G-x$ incident to the hinges $\{u, v\}$ and $\{w, z\}$, respectively. Then $S_{1}^{\prime}=\left\{u u_{1}, w w_{1}, e\right\}$ is a 3-edge-cut of $G$ and $X_{1}$ is a component of $G-S_{1}^{\prime}$. Using Claim 5, we deduce that $X_{1} \cong K_{1}$. Arguing similarly using $S_{2}^{\prime}=\left\{v v_{1}, z z_{1}, e\right\}$, we may deduce that $X_{2} \cong$ $K_{1}$. Thus $X \cong K_{2}^{3}$, contradicting the hypothesis that $X$ is 3-connected.

Claim 12 Let $X$ be a non-circuit cleavage unit of $G-x$. Then $X$ is a 3-bond.

Proof. Suppose $X$ is 3-connected. Let $T$ be the cleavage unit tree of $G-x$. By Claim 10(b), $X$ has degree two in $T$ and hence $X$ contains exactly two virtual edges $u_{1} v_{1}, w_{1} z_{1}$ of $G-x$. Let $S=\left\{u_{1} u_{2}, v_{1} v_{2}\right\}$ and $S^{\prime}=\left\{w_{1} w_{0}, z_{1} z_{0}\right\}$ be the 2-edge-cuts of $G-x$ incident to the hinges $\left\{u_{1}, v_{1}\right\}$ and $\left\{w_{1}, z_{1}\right\}$, respectively. Since $X$ is a 3 -connected cleavage unit of $G-x$, no vertex of $X$ is adjacent to $x$ in $G$.

Let $x_{1}, x_{2}, G^{-}, G^{+}$be as defined in the statement of Claim 9 with respect to $S$. By Lemma 2,

$$
F(G, t)=F\left(G^{+}, t\right)+F\left(G^{-}, t\right) .
$$

Let $G-S=H_{1} \cup H_{2}$ where $H_{1} \cap H_{2}=\{x\}$ and $V(X) \subseteq V\left(H_{1}\right)$. Let $G_{2}^{+}$be the graph obtained from $G^{+}$by contracting $E\left(H_{1}\right)$ to the single vertex $y_{2}$, and $G_{2}^{-}=$ $G_{2}^{+}-x_{2} y_{2}$. Let $G-S^{\prime}=H_{0} \cup H_{1}^{\prime}$ where $H_{0} \cap H_{1}^{\prime}=\{x\}$ and $V(X) \subseteq V\left(H_{1}^{\prime}\right)$. Define $G_{0}^{+}, G_{0}^{-}, y_{0}$ in a similar way to $G_{2}^{+}, G_{2}^{-}, y_{2}$ by contracting $E\left(H_{1}^{\prime}\right)$. Finally let $G_{1}^{-}$be obtained from $G^{-}$by contracting $E\left(H_{2}\right)$ to a single vertex $y_{2}$ and then contracting $E\left(H_{0}\right)$ to a single vertex $y_{0}$. Let $G_{1}^{+}=G_{1}^{-}+y_{2} y_{0}$. The 3-connectivity of $G$ impies that $G_{0}^{+}, G_{1}^{+}, G_{2}^{+}$are 3-connected, Claim 10(a) implies that $G_{2}^{-}, G_{0}^{-}$are essentially 3-connected, and the fact that $G_{1}^{-}$is homeomorphic to $X$ implies that $G_{1}^{-}$is essentially 3-connected.

Applying Lemma 7 twice to $G^{+}$using the 3-edge-cuts $\left\{u_{1} u_{2}, v_{1} v_{2}, x_{1} x_{2}\right\}$ and $\left\{w_{1} w_{0}, z_{1} z_{0}, x_{1} x_{2}\right\}$ we obtain

$$
(t-1)^{2}(t-2)^{2} F\left(G^{+}, t\right)=F\left(G_{0}^{+}, t\right) F\left(G_{1}^{+}, t\right) F\left(G_{2}^{+}, t\right) .
$$

Similarly, applying Lemma 5 twice to $G^{-}$using the 2-edge-cuts $\left\{u_{1} u_{2}, v_{1} v_{2}\right\}$ and $\left\{w_{1} w_{0}, z_{1} z_{0}\right\}$ we obtain

$$
(t-1)^{2} F\left(G^{-}, t\right)=F\left(G_{0}^{-}, t\right) F\left(G_{1}^{-}, t\right) F\left(G_{2}^{-}, t\right) .
$$


Substituting into (12) gives

$$
\begin{aligned}
(t-1)^{2}(t-2)^{2} F(G, t)= & F\left(G_{0}^{+}, t\right) F\left(G_{1}^{+}, t\right) F\left(G_{2}^{+}, t\right)+ \\
& (t-2)^{2} F\left(G_{0}^{-}, t\right) F\left(G_{1}^{-}, t\right) F\left(G_{2}^{-}, t\right) .
\end{aligned}
$$

Let $G_{1}$ be obtained from $G_{1}^{+}$by contracting the edge $y_{0} y_{2}$. Then $G_{1}$ is 3connected by Claim 11. By Lemma $2, F\left(G_{1}^{+}, t\right)=F\left(G_{1}, t\right)-F\left(G_{1}^{-}, t\right)$. Substituting into (13) we obtain

$$
\begin{aligned}
& (t-1)^{2}(t-2)^{2} F(G, t)=F\left(G_{0}^{+}, t\right) F\left(G_{2}^{+}, t\right) F\left(G_{1}, t\right)- \\
& \quad F\left(G_{1}^{-}, t\right)\left[F\left(G_{0}^{+}, t\right) F\left(G_{2}^{+}, t\right)-(t-2)^{2} F\left(G_{0}^{-}, t\right) F\left(G_{2}^{-}, t\right)\right] .
\end{aligned}
$$

We shall show that both of the terms on the right hand side of (14) are non-zero with sign $(-1)^{m-n+1}$.

Let $G_{3}$ be obtained from $G-V(X)$ by adding two new vertices $u, v$ and new edges $u v, u w_{0}, v z_{0}, u u_{2}, v v_{2}$. The 3-connectivity of $G$ implies that $G_{3}$ is 3-connected. We may apply the reduction used in the derivation of (13) to $G_{3}$. In this analysis the graphs $X, G_{1}^{+}$, and $G_{1}^{-}$are replaced by graphs isomorhic to $K_{2}^{3}, K_{4}$, and $K_{4}^{-}$, respectively, where $K_{4}^{-}$is obtained from $K_{4}$ by deleting an edge. Using the fact that $F\left(K_{4}, t\right)=(t-1)(t-2)(t-3)$ and $F\left(K_{4}^{-}, t\right)=(t-1)(t-2)$, we deduce that

$$
\begin{aligned}
(t-1)^{2}(t-2)^{2} F\left(G_{3}, t\right)= & F\left(G_{0}^{+}, t\right)(t-1)(t-2)(t-3) F\left(G_{2}^{+}, t\right)+ \\
& (t-2)^{2} F\left(G_{0}^{-}, t\right)(t-1)(t-2) F\left(G_{2}^{-}, t\right) .
\end{aligned}
$$

Applying induction to $G_{3}$ we have

$$
(-1)^{m_{3}-n_{3}+1}\left[(t-3) F\left(G_{0}^{+}, t\right) F\left(G_{2}^{+}, t\right)+(t-2)^{2} F\left(G_{0}^{-}, t\right) F\left(G_{2}^{-}, t\right)\right]>0 .
$$

Thus

$$
(-1)^{m_{3}-n_{3}}\left[(3-t) F\left(G_{0}^{+}, t\right) F\left(G_{2}^{+}, t\right)-(t-2)^{2} F\left(G_{0}^{-}, t\right) F\left(G_{2}^{-}, t\right)\right]>0 .
$$

By induction $(-1)^{m_{0}^{+}-n_{0}^{+}+m_{2}^{+}-n_{2}^{+}} F\left(G_{0}^{+}, t\right) F\left(G_{2}^{+}, t\right)>0$. Since $m_{3}=m_{0}^{+}+m_{2}^{+}-1$, $n_{3}=n_{0}^{+}+n_{2}^{+}-1$, and $3-t>0$, we have

$$
(-1)^{m_{3}-n_{3}}(3-t) F\left(G_{0}^{+}, t\right) F\left(G_{2}^{+}, t\right)>0 .
$$

Since $0<3-t<1$, it follows that

$$
(-1)^{m_{3}-n_{3}}\left[F\left(G_{0}^{+}, t\right) F\left(G_{2}^{+}, t\right)-(t-2)^{2} F\left(G_{0}^{-}, t\right) F\left(G_{2}^{-}, t\right)\right]>0 .
$$

Applying induction to $G_{1}^{-}$, we obtain $(-1)^{m_{1}^{-}-n_{1}^{-}+1} F\left(G_{1}^{-}, t\right)>0$. Using (15), and the facts that $m=m_{3}+m_{1}^{-}-5$, and $n=n_{3}+n_{1}^{-}-4$, we may deduce that

$$
(-1)^{m-n} F\left(G_{1}^{-}, t\right)\left[\left(F\left(G_{0}^{+}, t\right) F\left(G_{2}^{+}, t\right)-(t-2)^{2} F\left(G_{0}^{-}, t\right) F\left(G_{2}^{-}, t\right)\right]>0 .\right.
$$


We may also deduce by induction that

$$
(-1)^{m_{0}^{+}-n_{0}^{+}+m_{2}^{+}-n_{2}^{+}+m_{1}-n_{1}+1} F\left(G_{0}^{+}, t\right) F\left(G_{2}^{+}, t\right) F\left(G_{1}, t\right)>0 .
$$

Since $m=m_{0}^{+}+m_{2}^{+}+m_{1}-6$, and $n=n_{0}^{+}+n_{2}^{+}+n_{1}-4$, it follows that

$$
(-1)^{m-n+1} F\left(G_{0}^{+}, t\right) F\left(G_{2}^{+}, t\right) F\left(G_{1}, t\right)>0 .
$$

Using (14), (16) and (17), we deduce that $(-1)^{m-n+1} F(G, t)>0$. This contradicts the choice of $G$ as a counterexample to the theorem and completes the proof of the claim.

Claim 13 Let $X=v_{1} v_{2} \ldots v_{r} v_{1}$ be a circuit cleavage unit of $G-x$ which is not an end cleavage unit and $T$ be the cleavage unit tree of $G-x$.

(a) Each neighbour of $X$ in $T$ is a 3-bond cleavage unit of $G-x$.

(b) At most one vertex of $X$ is adjacent to $x$ in $G$.

(c) If a vertex of $X$ is adjacent to $x$ in $G$ then $X \cong C_{5}$ and $X$ has degree two in $T$.

(d) If no vertex of $X$ is adjacent to $x$ in $G$ then $X \cong C_{6}$ and $X$ has degree three in $T$.

Proof. (a) follows from Lemma 12(a) and Claim 12.

(b) Suppose $x v_{i}, x v_{j} \in E(G)$ for some $1 \leq i<j \leq r$. If $v_{j} \notin\left\{v_{i-1}, v_{i+1}\right\}$ then $S=\left\{v_{i-1} v_{i}, v_{j-1} v_{j}\right\}$ is a 2-edge-cut of $G-x$ which contradicts Claim 8(c). Hence we may assume without loss of generality that $x v_{1}, x v_{2} \in E(G)$ and that $x v_{s} \notin E(G)$ for all $3 \leq s \leq r$. Since each vertex of $G$ other than $x$ has degree 3, it follows that $r$ is even and $v_{2 s-1} v_{2 s}$ is a virtual edge of $G$ contained in $X$ for all $2 \leq s \leq r / 2$. Since $X$ has degree at least two in $T$, it contains at least two virtual edges of $G$ and hence $r \geq 6$. Let $S=\left\{v_{2} v_{3}, v_{r} v_{1}\right\}$. Then $S$ is a 2-edge-cut of $G-x$. Let $G^{-}, G_{1}^{-}, G_{2}^{-}$be as defined in the statement of Claim 8, where $v_{1}, v_{2} \in V\left(G_{1}^{-}\right)$. Since $G_{1}^{-}$s homeomorphic to $K_{2}^{3}, c_{1}^{-}=1$. Furthermore $c_{2}^{-}=1$ by Lemma 12(b). This contradicts Claim $8(\mathrm{a})$.

(c) We assume that $x$ is adjacent to exactly one vertex of $X$. Relabelling if necessary, we may assume without loss of generality that $x v_{1} \in E(G)$ and that $x v_{s} \notin E(G)$ for all $2 \leq s \leq r$. Thus $r$ is odd and $v_{2 s} v_{2 s+1}$ is a virtual edge of $G$ contained in $X$ for all $1 \leq s \leq(r-1) / 2$. Since $X$ has degree at least two in $G$, it contains at least two virtual edges of $G$ and hence $r \geq 5$. 
Suppose $r \geq 7$. Let $S=\left\{v_{1} v_{2}, v_{5} v_{6}\right\}$. Then $S$ is a 2-edge-cut of $G-x$. Let $G^{-}, G_{1}^{-}, G_{2}^{-}$be as defined in the statement of Claim 8, where $v_{1}, v_{6} \in V\left(G_{1}^{-}\right)$. Then $c_{1}^{-}=1$ by Claim $8(\mathrm{~b})$ and $c_{2}^{-}=1$ by Lemma 12(b). This contradicts Claim 8(a). Hence $r=5, X$ contains exactly two virtual edges of $G$, and $X$ has degree two in $T$.

(d) We assume that $x$ is adjacent to no vertex of $X$. Then $r$ is even and the virtual edges of $G$ in $X$ are a 1-factor of $X$. Relabelling if necessary, we may assume without loss of generality that $v_{2 s} v_{2 s+1}$ is a virtual edge of $G$ contained in $X$ for all $1 \leq s \leq r / 2$. Since $X$ has degree at least two in $G$, it contains at least two virtual edges of $G$ and hence $r \geq 4$.

Suppose $r=4$. Let $S=\left\{v_{1} v_{2}, v_{3} v_{4}\right\}$. Then $S$ is a 2-edge-cut of $G-x$. Let $G^{-}, G_{1}^{-}, G_{2}^{-}$be as defined in the statement of Claim 8, where $v_{1}, v_{4} \in V\left(G_{1}^{-}\right)$. Since the two cleavage units of $G-x$ adjacent to $X$ in $T$ have degree two in $T$ by Claim 10(b), we have $c_{1}^{-} \geq 2$ and $c_{2}^{-} \geq 2$. This gives a contradiction since Claim 8(a) and (d) imply that either $c_{1}^{-}=1$ or $c_{2}^{-}=1$. Hence $r \geq 6$.

Suppose $r \geq 8$. Let $S=\left\{v_{1} v_{2}, v_{5} v_{6}\right\}$. Then $S$ is a 2-edge-cut of $G-x$. Let $G^{-}, G_{1}^{-}, G_{2}^{-}$be as defined in the statement of Claim 8, where $v_{1}, v_{6} \in V\left(G_{1}^{-}\right)$. Then $c_{1}^{-}=1=c_{2}^{-}$by Lemma 12(b). This contradicts Claim 8(a). Hence $r=6, X$ contains exactly three virtual edges of $G$, and $X$ has degree three in $T$.

Lemma 21 and Claims 4, 9, 12, and 13 imply that $G$ is an extension of $\left(K_{4}, x\right)$. Since each single edge extension adds three vertices and five edges to $K_{4}$, we have $m-n$ is even. By Lemma $19, F(G, t)<0$ for $t \in(2, \alpha]$. This contradicts the choice of $G$ as a counterexample to the theorem.

Using Theorems 9, 22, and planar duality we have:

Corollary 23 Let $G$ be plane near triangulation. Then the only zeros of $P(G, t)$ in $(-\infty, \alpha]$ are 1 and 2 .

Since extensions of $\left(K_{4}, x\right)$ are planar, Theorem 20 and planar duality show that there exists an infinite family of plane near triangulations with chromatic roots converging to $\alpha$ from above.

\section{Closing Remarks}

In a subsequent paper [5] we extend the zero-free interval $(2, \alpha]$ for certain families of near-cubic graphs. As a corollary we deduce that if $G$ is a cubic bridgeless graph, then $F(G, t)$ has no zeros in $(2, \gamma)$, where $\gamma \approx 2.54 \ldots$ is the root in $(2,3)$ of 
the flow polynomial of the cube. The dual statement for plane triangulations had previously been verified by Woodall $[16,17]$.

Acknowledgement I would like to thank Douglas Woodall for his many helpful comments and suggestions. In particular for his suggestion that my original proof of Theorem 9 could be extended from flow polynomials to the derivatives of the function $q_{1}(G, t)$.

\section{References}

[1] G.D. Birkhoff and D.C. Lewis, Chromatic polynomials, Trans. Amer. Math. Soc. 60 (1946), 355-451.

[2] H. Edwards, R. Hierons and B. Jackson, The zero-free intervals for characteristic polynomials of matroids, Combinatorics, Probability and Computing 7 (1998), 153-165.

[3] B. Jackson, A zero-free interval for chromatic polynomials of graphs, Combinatorics, Probability and Computing 2 (1993), 325-336.

[4] B. Jackson, Zeros of chromatic and flow polynomials of graphs, J Geometry 76 (2003), 95-109.

[5] B. Jackson, A zero-free interval for flow polynomials of cubic graphs, submitted.

[6] F. Jaeger, Flows and generalized colouring theorems in graphs, J. Combinatorial Theory, Series B 26 (1979), 205-216.

[7] M. Kochal, Polynomials associated with nowhere-zero flows, J. Combinatorial Theory, Series B 84 (2002), 260-269.

[8] P.D. Seymour, Nowhere-zero 6-flows, J. Combinatorial Theory, Series B 30 (1981), 130-135.

[9] W.T. Tutte, On the imbedding of linear graphs in surfaces, Proc London Math. Soc. 51 (1950), 474-483.

[10] W.T. Tutte, A contribution to the theory of chromatic polynomials, Canad. J. Math. 6 (1954), 80-91.

[11] W.T. Tutte, Connectivity in Graphs, University of Toronto Press (1966). 
[12] W.T. Tutte, Chromials, Springer Lecture Notes in Mathematics 411 (1974), 243-266.

[13] C.D. Wakelin, Chromatic Polynomials, Ph.D. Thesis, University of Nottingham, (1994).

[14] D.J.A. Welsh, personal communication.

[15] D.R. Woodall, Zeros of chromatic polynomials, in Combinatorial Surveys (ed. P. Cameron), Proc. Sixth British Combinatorial Conference, Academic Press, London (1977), 199-223.

[16] D.R. Woodall, A zero-free interval for chromatic polynomials, Discrete Math. 101 (1992), 333-341.

[17] D.R. Woodall, Corrigendum A zero-free interval for chromatic polynomials, Discrete Math., to appear. 